\title{
A Sufficient Condition for Orbits of Hermann Actions to be Weakly Reflective
}

\author{
Shinji OHNO \\ Osaka City Universitiy Advanced Mathematical Institute \\ (Communicated by K. Ahara)
}

\begin{abstract}
In this paper, we give sufficient conditions for orbits of Hermann actions to be weakly reflective in terms of symmetric triads, that is a generalization of irreducible root systems. Using these sufficient conditions, we obtain new examples of weakly reflective submanifolds in compact symmetric spaces.
\end{abstract}

\section{Introduction}

Ikawa, Sakai, and Tasaki ([6]) proposed the notion of weakly reflective submanifold as a generalization of the notion of reflective submanifold ([8]). In [6], they detected a certain global symmetry of several austere submanifolds in a hypersphere, and classified austere orbits and weakly reflective orbits of the linear isotropy representation of irreducible symmetric spaces. They gave a necessary and sufficient condition for orbits of the linear isotropy representation of irreducible symmetric spaces to be an austere submanifold (further, weakly reflective submanifold) in the hypersphere in terms of root systems. We would like to generalize this fact to compact Riemannian symmetric spaces. However, it is known that austere orbits of the isotropy action of compact symmetric spaces are reflective submanifolds. Therefore, we consider Hermann actions which are a generalization of isotropy actions of compact symmetric spaces. Ikawa ([4]) introduced the notion of symmetric triad as a generalization of the notion of irreducible root system to study orbits of Hermann actions. Ikawa expressed orbit spaces of Hermann actions by using symmetric triads, and gave a characterization of the minimal, austere and totally geodesic orbits of Hermann actions in terms of symmetric triads. However, weakly reflective orbits have not been classified yet. In this paper, we give sufficient conditions for orbits of Hermann actions to be weakly reflective in terms of symmetric triads.

Let $G$ be a compact, connected, semisimple Lie group, and $K_{1}, K_{2}$ be symmetric subgroups of $G$. We consider the following three Lie group actions:

1. $\left(K_{2} \times K_{1}\right) \curvearrowright G:\left(k_{2}, k_{1}\right) g=k_{2} g k_{1}^{-1}\left(\left(k_{2}, k_{1}\right) \in K_{2} \times K_{1}\right)$,

2. $K_{2} \curvearrowright G / K_{1}: k_{2} \pi_{1}(g)=\pi_{1}\left(k_{2} g\right)\left(k_{2} \in K_{2}\right)$,

Received July 10, 2015; revised March 12, 2016

2010 Mathematics Subject Classification: 53C35 (Primary), 53C40 (Secondary)

Key words and phrases: Symmetric space, symmetric triad, root system, weakly reflective submanifold 
3. $K_{1} \curvearrowright K_{2} \backslash G: k_{1} \pi_{2}(g)=\pi_{2}\left(g k_{1}^{-1}\right)\left(k_{1} \in K_{1}\right)$.

The $K_{2}$-action and the $K_{1}$-action are called Hermann actions. Orbits of the $\left(K_{2} \times K_{1}\right)$ action have properties which are similar to orbits of Hermann actions. In particular, by using Ikawa's method, we characterize a minimal orbit and an austere orbit of the $\left(K_{2} \times K_{1}\right)$-action in terms of the symmetric triad determined by $\left(G, K_{1}, K_{2}\right)$. Since totally geodesic orbits of Hermann actions are reflective submanifolds, we only consider austere orbits which are not totally geodesic.

The organization of this paper is as follows. In Section 2, we prepare the foundation for the following sections. In 2.1, we recall the definition of weakly reflective submanifolds, and their main properties. In 2.2, we review the notion of root systems and symmetric triads. In particular, a minimal point, an austere point and a totally geodesic point are discussed. In Section 3, we express the second fundamental form of orbits of the $\left(K_{2} \times K_{1}\right)$-action on $G$ (Theorem 3), and characterize a minimal orbit and an austere orbit in terms of the symmetric triad of $\left(G, K_{1}, K_{2}\right)$ (Corollaries 2, 3). In Section 4, we give sufficient conditions for orbits of the above three group actions to be weakly reflective (Theorems 4, 5). Moreover, applying Theorem 5, we will construct new examples of weakly reflective submanifolds in compact symmetric spaces.

The author would like to thank O. Ikawa, T.Okuda and H. Tamaru for their useful advices.

\section{Preliminaries}

2.1. Weakly reflective submanifolds. We recall the definitions of reflective submanifold and weakly reflective submanifold. Let $(\tilde{M},\langle\rangle$,$) be a complete Riemannian manifold.$

Definition 1. Let $M$ be a submanifold of $\tilde{M}$. Then $M$ is a reflective submanifold of $\tilde{M}$ if there exists an involutive isometry $\sigma_{M}$ of $\tilde{M}$ such that $M$ is a connected component of the fixed point set of $\sigma_{M}$. Then, we call $\sigma_{M}$ the reflection of $M$.

Definition 2. Let $M$ be a submanifold of $\tilde{M}$. For each normal vector $\xi \in T_{x}^{\perp} M$ at each point $x \in M$, if there exists an isometry $\sigma_{\xi}$ on $\tilde{M}$ which satisfies $\sigma_{\xi}(x)=x, \sigma_{\xi}(M)=M$ and $\left(d \sigma_{\xi}\right)_{x}(\xi)=-\xi$, then we call $M$ a weakly reflective submanifold and $\sigma_{\xi}$ a reflection of $M$ with respect to $\xi$.

If $M$ is a reflective submanifold of $\tilde{M}$, then $\sigma_{M}$ is a reflection of $M$ with respect to each normal vector $\xi \in T_{x}^{\perp} M$ at each point $x \in M$. Thus, a reflective submanifold of $\tilde{M}$ is a weakly reflective submanifold of $\tilde{M}$. Notice that a reflective submanifold is totally geodesic, but a weakly reflective submanifold is not necessarily totally geodesic.

Definition 3 ([3]). Let $M$ be a submanifold of $\tilde{M}$. We denote the shape operator of $M$ by $A . M$ is called an austere submanifold if for each normal vector $\xi \in T_{x}^{\perp} M$, the set of eigenvalues with their multiplicities of $A^{\xi}$ is invariant under the multiplication by -1 . 
It is clear that an austere submanifold is a minimal submanifold. Ikawa, Sakai and Tasaki proved that a weakly reflective submanifold is an austere submanifold.

LEMMA 1 ([6]). Let $G$ be a Lie group acting isometrically on a Riemannian manifold $\tilde{M}$. For $x \in \tilde{M}$, we consider the orbit $G x$. If for each $\xi \in T_{x}^{\perp} G x$, there exists a reflection of $G x$ at $x$ with respect to $\xi$, then $G x$ is a weakly reflective submanifold of $\tilde{M}$.

PROPOSITION 1 ([6]). Any singular orbit of a cohomogeneity one action on a Riemannian manifold is a weakly reflective submanifold.

2.2. Symmetric triads. We recall the notions of root system and symmetric triad. See [4] for details.

Let $(\mathfrak{a},\langle\cdot, \cdot\rangle)$ be a finite dimensional inner product space over $\mathbf{R}$. For each $\alpha \in \mathfrak{a}$, we define an orthogonal transformation $s_{\alpha}: \mathfrak{a} \rightarrow \mathfrak{a}$ by

$$
s_{\alpha}(H)=H-\frac{2\langle\alpha, H\rangle}{\langle\alpha, \alpha\rangle} \alpha \quad(H \in \mathfrak{a}),
$$

namely $s_{\alpha}$ is the reflection with respect to the hyperplane $\{H \in \mathfrak{a} \mid\langle\alpha, H\rangle=0\}$.

Definition 4. A finite subset $\Sigma$ of $\mathfrak{a} \backslash\{0\}$ is a root system of $\mathfrak{a}$, if it satisfies the following three conditions:

1. $\operatorname{Span}(\Sigma)=\mathfrak{a}$.

2. If $\alpha, \beta \in \Sigma$, then $s_{\alpha}(\beta) \in \Sigma$.

3. $2\langle\alpha, \beta\rangle /\langle\alpha, \alpha\rangle \in \mathbf{Z} \quad(\alpha, \beta \in \Sigma)$.

A root system of $\mathfrak{a}$ is said to be irreducible if it cannot be decomposed into two disjoint nonempty orthogonal subsets.

Let $\Sigma$ be a root system of $\mathfrak{a}$. The Weyl group $W(\Sigma)$ of $\Sigma$ is the finite subgroup of the orthogonal group $\mathrm{O}(\mathfrak{a})$ of a generated by $\left\{s_{\alpha} \mid \alpha \in \Sigma\right\}$.

Definition 5 ([4] Definition 2.2). A triple $(\tilde{\Sigma}, \Sigma, W)$ of finite subsets of $\mathfrak{a} \backslash\{0\}$ is a symmetric triad of $\mathfrak{a}$, if it satisfies the following six conditions:

1. $\tilde{\Sigma}$ is an irreducible root system of $\mathfrak{a}$.

2. $\Sigma$ is a root system of $\mathfrak{a}$.

3. $(-1) W=W, \tilde{\Sigma}=\Sigma \cup W$.

4. $\Sigma \cap W$ is a nonempty subset. If we put $l:=\max \{\|\alpha\| \mid \alpha \in \Sigma \cap W\}$, then $\Sigma \cap W=$ $\{\alpha \in \tilde{\Sigma} \mid\|\alpha\| \leq l\}$.

5. For $\alpha \in W$ and $\lambda \in \Sigma \backslash W$,

$$
2 \frac{\langle\alpha, \lambda\rangle}{\langle\alpha, \alpha\rangle} \text { is odd if and only if } s_{\alpha}(\lambda) \in W \backslash \Sigma .
$$


6. For $\alpha \in W$ and $\lambda \in W \backslash \Sigma$,

$$
2 \frac{\langle\alpha, \lambda\rangle}{\langle\alpha, \alpha\rangle} \text { is odd if and only if } s_{\alpha}(\lambda) \in \Sigma \backslash W .
$$

Let $(\tilde{\Sigma}, \Sigma, W)$ be a symmetric triad of $\mathfrak{a}$. We set

$$
\begin{aligned}
& \Gamma=\{H \in \mathfrak{a} \mid\langle\lambda, H\rangle \in(\pi / 2) \mathbf{Z} \quad(\lambda \in \tilde{\Sigma})\}, \\
& \Gamma_{\Sigma \cap W}=\{H \in \mathfrak{a} \mid\langle\lambda, H\rangle \in(\pi / 2) \mathbf{Z} \quad(\lambda \in \Sigma \cap W)\} .
\end{aligned}
$$

A point in $\Gamma$ is called a totally geodesic point. It is known that $\Gamma=\Gamma_{\Sigma \cap W}$. We define an open subset $\mathfrak{a}_{r}$ of $\mathfrak{a}$ by

$$
\mathfrak{a}_{r}=\bigcap_{\lambda \in \Sigma, \alpha \in W}\left\{H \in \mathfrak{a} \mid\langle\lambda, H\rangle \notin \pi \mathbf{Z},\langle\alpha, H\rangle \notin \frac{\pi}{2}+\pi \mathbf{Z}\right\} .
$$

A point in $\mathfrak{a}_{r}$ is called a regular point, and a point in the complement of $\mathfrak{a}_{r}$ in $\mathfrak{a}$ is called a singular point. A connected component of $\mathfrak{a}_{r}$ is called a cell. The affine Weyl group $\tilde{W}(\tilde{\Sigma}, \Sigma, W)$ of $(\tilde{\Sigma}, \Sigma, W)$ is a subgroup of the affine group of $\mathfrak{a}$, which defined by the semidirect product $\mathrm{O}(\mathfrak{a}) \ltimes \mathfrak{a}$, generated by

$$
\left\{\left(s_{\lambda}, \frac{2 n \pi}{\langle\lambda, \lambda\rangle} \lambda\right) \mid \lambda \in \Sigma, n \in \mathbf{Z}\right\} \cup\left\{\left(s_{\alpha}, \frac{(2 n+1) \pi}{\langle\alpha, \alpha\rangle} \alpha\right) \mid \alpha \in W, n \in \mathbf{Z}\right\} .
$$

The action of $\left(s_{\lambda},(2 n \pi /\langle\lambda, \lambda\rangle) \lambda\right)$ on $\mathfrak{a}$ is the reflection with respect to the hyperplane $\{H \in \mathfrak{a} \mid$ $\langle\lambda, H\rangle=n \pi\}$, and the action of $\left(s_{\alpha},((2 n+1) \pi /\langle\alpha, \alpha\rangle) \alpha\right)$ on $\mathfrak{a}$ is the reflection with respect to the hyperplane $\{H \in \mathfrak{a} \mid\langle\alpha, H\rangle=((2 n+1) / 2) \pi\}$. The affine Weyl group $\tilde{W}(\tilde{\Sigma}, \Sigma, W)$ acts transitively on the set of all cells. More precisely, for each cell $P$, it holds that

$$
\mathfrak{a}=\bigcup_{s \in \tilde{W}(\tilde{\Sigma}, \Sigma, W)} s \bar{P} .
$$

We take a fundamental system $\tilde{\Pi}$ of $\tilde{\Sigma}$. We denote by $\tilde{\Sigma}^{+}$the set of positive roots in $\tilde{\Sigma}$. Set $\Sigma^{+}=\tilde{\Sigma}^{+} \cap \Sigma$ and $W^{+}=\tilde{\Sigma}^{+} \cap W$. Denote by $\Pi$ the set of simple roots of $\Sigma$. We set

$$
W_{0}=\left\{\alpha \in W^{+} \mid \alpha+\lambda \notin W(\lambda \in \Pi)\right\} .
$$

From the classification of symmetric triads, we have that $W_{0}$ consists of the only one element, denoted by $\tilde{\alpha}$. We define an open subset $P_{0}$ of $\mathfrak{a}$ by

$$
P_{0}=\left\{H \in \mathfrak{a} \mid\langle\tilde{\alpha}, H\rangle<\frac{\pi}{2},\langle\lambda, H\rangle>0(\lambda \in \Pi)\right\} .
$$


Then $P_{0}$ is a cell. For a nonempty subset $\Delta \subset \Pi \cup\{\tilde{\alpha}\}$, set

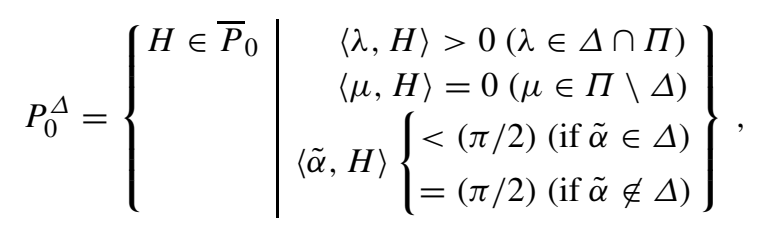

then

$$
\bar{P}_{0}=\bigcup_{\Delta \subset \Pi \cup\{\tilde{\alpha}\}} P_{0}^{\Delta} \text { (disjoint union) } .
$$

Definition 6 ([4] Definition 2.13). Let $(\tilde{\Sigma}, \Sigma, W)$ be a symmetric triad of $\mathfrak{a}$. Consider two mappings $m$ and $n$ from $\tilde{\Sigma}$ to $\mathbf{R}_{\geq 0}:=\{a \in \mathbf{R} \mid a \geq 0\}$ which satisfy the following four conditions:

1. For any $\lambda \in \tilde{\Sigma}$,

(1-1) $m(\lambda)=m(-\lambda), n(\lambda)=n(-\lambda)$,

(1-2) $m(\lambda)>0$ if and only if $\lambda \in \Sigma$,

(1-3) $n(\lambda)>0$ if and only if $\lambda \in W$.

2. When $\lambda \in \Sigma, \alpha \in W, s \in W(\Sigma)$, then $m(\lambda)=m(s(\lambda)), n(\alpha)=n(s(\alpha))$.

3. When $\lambda \in \tilde{\Sigma}, \sigma \in W(\tilde{\Sigma})$, then $m(\lambda)+n(\lambda)=m(\sigma(\lambda))+n(\sigma(\lambda))$.

4. Let $\lambda \in \Sigma \cap W, \alpha \in W$. If $2\langle\alpha, \lambda\rangle /\langle\alpha, \alpha\rangle$ is even, then $m(\lambda)=m\left(s_{\alpha}(\lambda)\right)$. If $2\langle\alpha, \lambda\rangle /\langle\alpha, \alpha\rangle$ is odd, then $m(\lambda)=n\left(s_{\alpha}(\lambda)\right)$.

We call $m(\lambda)$ and $n(\alpha)$ the multiplicities of $\lambda$ and $\alpha$, respectively.

Let $(\tilde{\Sigma}, \Sigma, W)$ be a symmetric triad of $\mathfrak{a}$ with multiplicities $m$ and $n$. For $H \in \mathfrak{a}$, we set

$$
m_{H}=-\sum_{\substack{\lambda \in \Sigma^{+} \\\langle\lambda, H\rangle \notin \pi \mathbf{Z}}} m(\lambda) \cot \langle\lambda, H\rangle \lambda+\sum_{\substack{\alpha \in W^{+} \\\langle\alpha, H\rangle \notin(\pi / 2)+\pi \mathbf{Z}}} n(\alpha) \tan \langle\alpha, H\rangle \alpha .
$$

The vector $m_{H}$ is called the mean curvature vector at $H$. A vector $H \in \mathfrak{a}$ is a minimal point if $m_{H}=0$.

Proposition 2 (Theorem 2.14 in [4]). Let $(\tilde{\Sigma}, \Sigma, W)$ be a symmetric triad of $\mathfrak{a}$ with multiplicities. For $H \in \mathfrak{a}$ and $\sigma=(s, X) \in \tilde{W}(\tilde{\Sigma}, \Sigma, W)$, set $H^{\prime}=\sigma H \in \mathfrak{a}$, then

$$
m_{H^{\prime}}=s\left(m_{H}\right) \text {. }
$$

THEOREM 1 (Theorem 2.24 in [4]). For any nonempty subset $\Delta \subset \Pi \cup\{\tilde{\alpha}\}$, there exists a unique minimal point $H \in P_{0}^{\Delta}$.

A vector $H \in \mathfrak{a}$ is an austere point if the subset of $\mathfrak{a}$ with multiplicities defined by

$$
\left.\{-\cot \langle\lambda, H\rangle \lambda \text { (multiplicity }=m(\lambda)) \mid \lambda \in \Sigma^{+},\langle\lambda, H\rangle \notin \pi \mathbf{Z}\right\}
$$




$$
\left.\cup\{\tan \langle\alpha, H\rangle \alpha \text { (multiplicity }=n(\alpha)) \mid \alpha \in W^{+},\langle\alpha, H\rangle \notin(\pi / 2)+\pi \mathbf{Z}\right\}
$$

is invariant with multiplicities under the multiplication by -1 . An austere point is a minimal point.

Proposition 3 ([4] Theorem 2.18). A point $H \in \mathfrak{a}$ is austere if and only if the following three conditions holds:

1. $\langle\lambda, H\rangle \in(\pi / 2) \mathbf{Z}$ for any $\lambda \in(\Sigma \backslash W) \cup(W \backslash \Sigma)$.

2. $2 H \in \Gamma_{\Sigma \cap W}$.

3. $m(\lambda)=n(\lambda)$ for any $\lambda \in \Sigma \cap W$ with $\langle\lambda, H\rangle \in(\pi / 4)+(\pi / 2) \mathbf{Z}$.

Ikawa gave the classification of symmetric triad and determined austere points for symmetric triads with multiplicities.

\section{Minimal orbits and austere orbits}

In this section, we consider Hermann actions and associated actions on Lie groups which are hyperpolar actions on compact symmetric spaces. An isometric action of a compact Lie group on a Riemannian manifold $M$ is called hyperpolar if there exists a closed, connected and flat submanifold $S$ of $M$ that meets all orbits orthogonally. Then, the submanifold $S$ is called a section. A. Kollross ([7]) classified the hyperpolar actions on compact irreducible symmetric spaces. By the classification, we can see that a hyperpolar action on a compact symmetric space whose cohomogeneity is two or greater, is orbit-equivalent to some Hermann action.

Let $G$ be a compact, connected, semisimple Lie group, and $K_{1}, K_{2}$ be closed subgroups of $G$. For each $i=1,2$, assume that there exists an involutive automorphism $\theta_{i}$ of $G$ which satisfies $\left(G_{\theta_{i}}\right)_{0} \subset K_{i} \subset G_{\theta_{i}}$, where $G_{\theta_{i}}$ is the set of fixed points of $\theta_{i}$ and $\left(G_{i}\right)_{0}$ is the identity component of $G_{\theta_{i}}$. Then the triple $\left(G, K_{1}, K_{2}\right)$ is called a compact symmetric triad. The pair $\left(G, K_{i}\right)$ is a compact symmetric pair for $i=1,2$. We denote the Lie algebras of $G, K_{1}$ and $K_{2}$ by $\mathfrak{g}, \mathfrak{k}_{1}$ and $\mathfrak{k}_{2}$, respectively. The involutive automorphism of $\mathfrak{g}$ induced from $\theta_{i}$ will be also denoted by $\theta_{i}$. Take an $\operatorname{Ad}(G)$-invariant inner product $\langle\cdot, \cdot\rangle$ on $\mathfrak{g}$. Then the inner product $\langle\cdot, \cdot\rangle$ induces a bi-invariant Riemannian metric on $G$ and $G$-invariant Riemannian metrics on the coset manifolds $M_{1}:=G / K_{1}$ and $M_{2}:=K_{2} \backslash G$. We denote these Riemannian metrics on $G, M_{1}$ and $M_{2}$ by the same symbol $\langle\cdot, \cdot\rangle$. These Riemannian manifolds $G, M_{1}$ and $M_{2}$ are Riemannian symmetric spaces with respect to $\langle\cdot, \cdot\rangle$. We denote by $\pi_{i}$ the natural projection from $G$ to $M_{i}(i=1,2)$, and consider the following three Lie group actions:

- $\left(K_{2} \times K_{1}\right) \curvearrowright G:\left(k_{2}, k_{1}\right) g=k_{2} g k_{1}^{-1}\left(\left(k_{2}, k_{1}\right) \in K_{2} \times K_{1}\right)$,

- $K_{2} \curvearrowright M_{1}: k_{2} \pi_{1}(g)=\pi_{1}\left(k_{2} g\right) \quad\left(k_{2} \in K_{2}\right)$,

- $K_{1} \curvearrowright M_{2}: k_{1} \pi_{2}(g)=\pi_{2}\left(g k_{1}^{-1}\right)\left(k_{1} \in K_{1}\right)$,

for $g \in G$. The three actions have the same orbit space, and in fact, the following diagram is 
commutative:

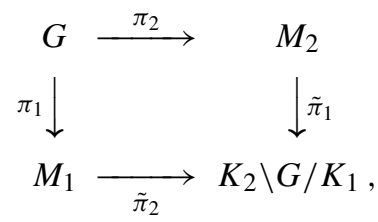

where $\tilde{\pi}_{i}$ is the natural projection from $M_{i}$ to the orbit space $K_{2} \backslash G / K_{1}$. Ikawa computed the second fundamental form of orbits of Hermann actions in the case $\theta_{1} \theta_{2}=\theta_{2} \theta_{1}$. We can apply Ikawa's method to the geometry of orbits of the $\left(K_{2} \times K_{1}\right)$-action. For $g \in G$, we denote the left (resp. right) transformation of $G$ by $L_{g}$ (resp. $R_{g}$ ). The isometry on $M_{1}$ (resp. $M_{2}$ ) induced by $L_{g}$ (resp. $R_{g}$ ) will be also denoted by the same symbol $L_{g}$ (resp. $R_{g}$ ).

For $i=1,2$, we set

$$
\mathfrak{m}_{i}=\left\{X \in \mathfrak{g} \mid \theta_{i}(X)=-X\right\} .
$$

Then we have an orthogonal direct sum decomposition of $\mathfrak{g}$ that is the canonical decomposition:

$$
\mathfrak{g}=\mathfrak{k}_{i} \oplus \mathfrak{m}_{i} .
$$

Let $e$ denotes the identity element of $G$. The tangent space $T_{\pi_{i}(e)} M_{i}$ of $M_{i}$ at the origin $\pi_{i}(e)$ is identified with $\mathfrak{m}_{i}$ in a natural way. We define a closed subgroup $G_{12}$ of $G$ by

$$
G_{12}=\left\{g \in G \mid \theta_{1}(g)=\theta_{2}(g)\right\} .
$$

Hence $\left(\left(G_{12}\right)_{0}, K_{12}\right)$ is a compact symmetric pair, where $K_{12}$ is a closed subgroup of $\left(G_{12}\right)_{0}$ defined by

$$
K_{12}=\left\{k \in\left(G_{12}\right)_{0} \mid \theta_{1}(k)=k\right\} .
$$

The canonical decomposition of $\left(\left(G_{12}\right)_{0}, K_{12}\right)$ is given by

$$
\mathfrak{g}_{12}=\left(\mathfrak{k}_{1} \cap \mathfrak{k}_{2}\right) \oplus\left(\mathfrak{m}_{1} \cap \mathfrak{m}_{2}\right) .
$$

Fix a maximal abelian subspace $\mathfrak{a}$ in $\mathfrak{m}_{1} \cap \mathfrak{m}_{2}$. Then $\exp (\mathfrak{a})$ is a toral subgroup in $\left(G_{12}\right)_{0}$. Then $\exp (\mathfrak{a}), \pi_{1}(\exp (\mathfrak{a}))$ and $\pi_{2}(\exp (\mathfrak{a}))$ are sections of the $\left(K_{2} \times K_{1}\right)$-action, the $K_{2}$-action and the $K_{1}$-action, respectively. To investigate the orbit spaces of the three actions, we consider an equivalent relation $\sim$ on $\mathfrak{a}$ defined as follows: For $H_{1}, H_{2} \in \mathfrak{a}, H_{1} \sim H_{2}$ if $K_{2} \exp \left(H_{1}\right) K_{1}=$ $K_{2} \exp \left(H_{2}\right) K_{1}$. Clearly, we have $H_{1} \sim H_{2}$ if and only if $K_{2} \pi_{1}\left(\exp \left(H_{1}\right)\right)=K_{2} \pi_{1}\left(\exp \left(H_{2}\right)\right)$, and similarly, $H_{1} \sim H_{2}$ if and only if $K_{1} \pi_{2}\left(\exp \left(H_{1}\right)\right)=K_{1} \pi_{2}\left(\exp \left(H_{2}\right)\right)$. Then we have $\mathfrak{a} / \sim=K_{2} \backslash G / K_{1}$. For each subgroup $L$ of $G$, we define

$$
\begin{aligned}
& N_{L}(\mathfrak{a})=\{k \in L \mid \operatorname{Ad}(k) \mathfrak{a}=\mathfrak{a}\}, \\
& Z_{L}(\mathfrak{a})=\{k \in L \mid \operatorname{Ad}(k) H=H(H \in \mathfrak{a})\} .
\end{aligned}
$$


Then $Z_{L}(\mathfrak{a})$ is a normal subgroup of $N_{L}(\mathfrak{a})$. We define a group $\tilde{J}$ by

$$
\tilde{J}=\left\{([s], Y) \in N_{K_{2}}(\mathfrak{a}) / Z_{K_{1} \cap K_{2}}(\mathfrak{a}) \ltimes \mathfrak{a} \mid \exp (-Y) s \in K_{1}\right\} .
$$

The group $\tilde{J}$ naturally acts on $\mathfrak{a}$ by the following:

$$
([s], Y) H=\operatorname{Ad}(s) H+Y(([s], Y) \in \tilde{J}, H \in \mathfrak{a}) .
$$

Matsuki ([9]) proved that

$$
K_{2} \backslash G / K_{1} \cong \mathfrak{a} / \tilde{J}
$$

Hereafter, we suppose $\theta_{1} \theta_{2}=\theta_{2} \theta_{1}$. Then we have an orthogonal direct sum decomposition of $\mathfrak{g}$ :

$$
\mathfrak{g}=\left(\mathfrak{k}_{1} \cap \mathfrak{k}_{2}\right) \oplus\left(\mathfrak{m}_{1} \cap \mathfrak{m}_{2}\right) \oplus\left(\mathfrak{k}_{1} \cap \mathfrak{m}_{2}\right) \oplus\left(\mathfrak{m}_{1} \cap \mathfrak{k}_{2}\right) .
$$

We define subspaces of $\mathfrak{g}$ as follows:

$$
\begin{aligned}
\mathfrak{k}_{0} & =\left\{X \in \mathfrak{k}_{1} \cap \mathfrak{k}_{2} \mid[\mathfrak{a}, X]=\{0\}\right\}, \\
V\left(\mathfrak{k}_{1} \cap \mathfrak{m}_{2}\right) & =\left\{X \in \mathfrak{k}_{1} \cap \mathfrak{m}_{2} \mid[\mathfrak{a}, X]=\{0\}\right\}, \\
V\left(\mathfrak{m}_{1} \cap \mathfrak{k}_{2}\right) & =\left\{X \in \mathfrak{m}_{1} \cap \mathfrak{k}_{2} \mid[\mathfrak{a}, X]=\{0\}\right\} .
\end{aligned}
$$

For $\lambda \in \mathfrak{a}$,

$$
\begin{aligned}
\mathfrak{k}_{\lambda} & =\left\{X \in \mathfrak{k}_{1} \cap \mathfrak{k}_{2} \mid[H,[H, X]]=-\langle\lambda, H\rangle^{2} X(H \in \mathfrak{a})\right\}, \\
\mathfrak{m}_{\lambda} & =\left\{X \in \mathfrak{m}_{1} \cap \mathfrak{m}_{2} \mid[H,[H, X]]=-\langle\lambda, H\rangle^{2} X(H \in \mathfrak{a})\right\}, \\
V_{\lambda}^{\perp}\left(\mathfrak{k}_{1} \cap \mathfrak{m}_{2}\right) & =\left\{X \in \mathfrak{k}_{1} \cap \mathfrak{m}_{2} \mid[H,[H, X]]=-\langle\lambda, H\rangle^{2} X(H \in \mathfrak{a})\right\}, \\
V_{\lambda}^{\perp}\left(\mathfrak{m}_{1} \cap \mathfrak{k}_{2}\right) & =\left\{X \in \mathfrak{m}_{1} \cap \mathfrak{k}_{2} \mid[H,[H, X]]=-\langle\lambda, H\rangle^{2} X(H \in \mathfrak{a})\right\} .
\end{aligned}
$$

We set

$$
\begin{aligned}
\Sigma & =\left\{\lambda \in \mathfrak{a} \backslash\{0\} \mid \mathfrak{k}_{\lambda} \neq\{0\}\right\}, \\
W & =\left\{\alpha \in \mathfrak{a} \backslash\{0\} \mid V_{\alpha}^{\perp}\left(\mathfrak{k}_{1} \cap \mathfrak{m}_{2}\right) \neq\{0\}\right\}, \\
\tilde{\Sigma} & =\Sigma \cup W .
\end{aligned}
$$

It is known that $\operatorname{dim} \mathfrak{k}_{\lambda}=\operatorname{dim} \mathfrak{m}_{\lambda}$ and $\operatorname{dim} V_{\lambda}^{\perp}\left(\mathfrak{k}_{1} \cap \mathfrak{m}_{2}\right)=\operatorname{dim} V_{\lambda}^{\perp}\left(\mathfrak{m}_{1} \cap \mathfrak{k}_{2}\right)$ for each $\lambda \in \tilde{\Sigma}$. Thus we set $m(\lambda):=\operatorname{dim} \mathfrak{k}_{\lambda}, n(\lambda):=\operatorname{dim} V_{\lambda}^{\perp}\left(\mathfrak{k}_{1} \cap \mathfrak{m}_{2}\right)$. Notice that $\Sigma$ is the root system of the pair $\left(\left(G_{12}\right)_{0}, K_{12}\right)$, and $\tilde{\Sigma}$ is a root system of $\mathfrak{a}$ (see [4]). We take a basis of $\mathfrak{a}$ and the lexicographic ordering $>$ on $\mathfrak{a}$ with respect to the basis. We set

$$
\tilde{\Sigma}^{+}=\{\lambda \in \tilde{\Sigma} \mid \lambda>0\}, \Sigma^{+}=\Sigma \cap \tilde{\Sigma}^{+}, W^{+}=W \cap \tilde{\Sigma}^{+} .
$$

Then we have an orthogonal direct sum decomposition of $\mathfrak{g}$ : 


$$
\begin{array}{r}
\mathfrak{g}=\mathfrak{k}_{0} \oplus \sum_{\lambda \in \Sigma^{+}} \mathfrak{k}_{\lambda} \oplus \mathfrak{a} \oplus \sum_{\lambda \in \Sigma^{+}} \mathfrak{m}_{\lambda} \oplus V\left(\mathfrak{k}_{1} \cap \mathfrak{m}_{2}\right) \oplus \sum_{\alpha \in W^{+}} V_{\alpha}^{\perp}\left(\mathfrak{k}_{1} \cap \mathfrak{m}_{2}\right) \\
\oplus V\left(\mathfrak{m}_{1} \cap \mathfrak{k}_{2}\right) \oplus \sum_{\alpha \in W^{+}} V_{\alpha}^{\perp}\left(\mathfrak{m}_{1} \cap \mathfrak{k}_{2}\right) .
\end{array}
$$

Furthermore, we have the following lemma.

Lemma 2 ([4] Lemmas 4.3 and 4.16). $\quad$ 1. For each $\lambda \in \Sigma^{+}$, there exist orthonormal bases $\left\{S_{\lambda, i}\right\}_{1 \leq i \leq m(\lambda)}$ and $\left\{T_{\lambda, i}\right\}_{1 \leq i \leq m(\lambda)}$ of $\mathfrak{k}_{\lambda}$ and $\mathfrak{m}_{\lambda}$ respectively such that for any $H \in \mathfrak{a}$,

$$
\begin{gathered}
{\left[H, S_{\lambda, i}\right]=\langle\lambda, H\rangle T_{\lambda, i}, \quad\left[H, T_{\lambda, i}\right]=-\langle\lambda, H\rangle S_{\lambda, i}, \quad\left[S_{\lambda, i}, T_{\lambda, i}\right]=\lambda,} \\
\operatorname{Ad}(\exp H) S_{\lambda, i}=\cos \langle\lambda, H\rangle S_{\lambda, i}+\sin \langle\lambda, H\rangle T_{\lambda, i}, \\
\operatorname{Ad}(\exp H) T_{\lambda, i}=-\sin \langle\lambda, H\rangle S_{\lambda, i}+\cos \langle\lambda, H\rangle T_{\lambda, i} .
\end{gathered}
$$

2. For each $\alpha \in W^{+}$, there exist orthonormal bases $\left\{X_{\alpha, j}\right\}_{1 \leq j \leq n(\alpha)}$ and $\left\{Y_{\alpha, j}\right\}_{1 \leq j \leq n(\alpha)}$ of $V_{\alpha}^{\perp}\left(\mathfrak{k}_{1} \cap \mathfrak{m}_{2}\right)$ and $V_{\alpha}^{\perp}\left(\mathfrak{m}_{1} \cap \mathfrak{k}_{2}\right)$ respectively such that for any $H \in \mathfrak{a}$

$$
\begin{aligned}
{\left[H, X_{\alpha, j}\right]=} & \langle\alpha, H\rangle Y_{\alpha, j}, \quad\left[H, Y_{\alpha, j}\right]=-\langle\alpha, H\rangle X_{\alpha, j}, \quad\left[X_{\alpha, j}, Y_{\alpha, j}\right]=\alpha, \\
& \operatorname{Ad}(\exp H) X_{\alpha, j}=\cos \langle\alpha, H\rangle X_{\alpha, j}+\sin \langle\alpha, H\rangle Y_{\alpha, j}, \\
& \operatorname{Ad}(\exp H) Y_{\alpha, j}=-\sin \langle\alpha, H\rangle X_{\alpha, j}+\cos \langle\alpha, H\rangle Y_{\alpha, j} .
\end{aligned}
$$

Using Lemma 2, Ikawa proved the following theorem.

TheOREM 2 ([4] Corollaries 4.23, 4.29, 4.24, and [2] Theorem 5.3). Let $g=\exp (H)$ $(H \in \mathfrak{a})$. Denote the mean curvature vector of $K_{2} \pi_{1}(g) \subset M_{1}$ at $\pi_{1}(g)$ by $m_{H}^{1}$. Then we have:

(1)

$$
d L_{g}^{-1} m_{H}^{1}=-\sum_{\substack{\lambda \in \Sigma^{+} \\\langle\lambda, H\rangle \notin \pi \mathbf{Z}}} m(\lambda) \cot \langle\lambda, H\rangle \lambda+\sum_{\substack{\alpha \in W^{+} \\\langle\alpha, H\rangle \notin(\pi / 2)+\pi \mathbf{Z}}} n(\alpha) \tan \langle\alpha, H\rangle \alpha .
$$

(2) The orbit $K_{2} \pi_{1}(g) \subset M_{1}$ is austere if and only if the finite subset of $\mathfrak{a}$ defined by

$$
\begin{gathered}
\left.\{-\lambda \cot \langle\lambda, H\rangle \text { (multiplicity }=m(\lambda)) \mid \lambda \in \Sigma^{+},\langle\lambda, H\rangle \notin \pi \mathbf{Z}\right\} \\
\left.\cup\{\alpha \tan \langle\alpha, H\rangle \text { (multiplicity }=n(\alpha)) \mid \alpha \in W^{+},\langle\alpha, H\rangle \notin(\pi / 2)+\pi \mathbf{Z}\right\}
\end{gathered}
$$

is invariant under the multiplication by -1 with multiplicities.

(3) The orbit $K_{2} \pi_{1}(g) \subset M_{1}$ is totally geodesic if and only if $\langle\lambda, H\rangle \in(\pi / 2) \mathbf{Z}$ for each $\lambda \in \tilde{\Sigma}^{+}$. 
We can apply Theorem 2 for orbits $K_{1} \pi_{2}(g) \subset M_{2}$. Thus, we have the following corollary.

COROLlaRY 1. The orbit $K_{2} \pi_{1}(g)$ is minimal (resp. austere, totally geodesic) if and only if $K_{1} \pi_{2}(g)$ is minimal (resp. austere, totally geodesic).

Now we consider the second fundamental form of orbits of the $\left(K_{2} \times K_{1}\right)$-action on $G$. For $H \in \mathfrak{a}$, we set

$$
\begin{aligned}
& \Sigma_{H}=\{\lambda \in \Sigma \mid\langle\lambda, H\rangle \in \pi \mathbf{Z}\}, W_{H}=\{\alpha \in W \mid\langle\alpha, H\rangle \in(\pi / 2)+\pi \mathbf{Z}\}, \\
& \tilde{\Sigma}_{H}=\Sigma_{H} \cup W_{H}, \Sigma_{H}^{+}=\Sigma^{+} \cap \Sigma_{H}, W_{H}^{+}=W^{+} \cap W_{H}, \tilde{\Sigma}_{H}^{+}=\Sigma_{H}^{+} \cup W_{H}^{+} .
\end{aligned}
$$

Let $g=\exp (H)(H \in \mathfrak{a})$. Then we have

$$
\begin{aligned}
& T_{g}\left(K_{2} g K_{1}\right)=\left\{\left.\frac{d}{d t} \exp \left(t X_{2}\right) g \exp \left(-t X_{1}\right)\right|_{t=0} \mid X_{1} \in \mathfrak{k}_{1}, X_{2} \in \mathfrak{k}_{2}\right\} \\
&= d L_{g}\left(\left(\operatorname{Ad}(g)^{-1} \mathfrak{k}_{2}\right)+\mathfrak{k}_{1}\right) \\
&= d L_{g}\left(\mathfrak{k}_{0} \oplus V\left(\mathfrak{m}_{1} \cap \mathfrak{k}_{2}\right) \oplus \sum_{\lambda \in \Sigma^{+} \backslash \Sigma_{H}} \mathfrak{m}_{\lambda} \oplus \sum_{\alpha \in W^{+} \backslash W_{H}} V_{\alpha}^{\perp}\left(\mathfrak{m}_{1} \cap \mathfrak{k}_{2}\right)\right. \\
&\left.\oplus V\left(\mathfrak{k}_{1} \cap \mathfrak{m}_{2}\right) \oplus \sum_{\lambda \in \Sigma^{+}} \mathfrak{k}_{\lambda} \oplus \sum_{\alpha \in W^{+}} V_{\alpha}^{\perp}\left(\mathfrak{k}_{1} \cap \mathfrak{m}_{2}\right)\right), \\
& T_{g}^{\perp}\left(K_{2} g K_{1}\right)=d L_{g}\left(\left(\operatorname{Ad}(g)^{-1} \mathfrak{m}_{2}\right) \cap \mathfrak{m}_{1}\right) \\
&=d L_{g}\left(\mathfrak{a} \oplus \sum_{\lambda \in \Sigma_{H}^{+}} \mathfrak{m}_{\lambda} \oplus \sum_{\alpha \in W_{H}^{+}} V_{\alpha}^{\perp}\left(\mathfrak{m}_{1} \cap \mathfrak{k}_{2}\right)\right) .
\end{aligned}
$$

For $X=\left(X_{2}, X_{1}\right) \in \mathfrak{g} \times \mathfrak{g}$, we define a Killing vector field $X^{*}$ on $G$ by

$$
\left(X^{*}\right)_{p}=\left.\frac{d}{d t} \exp \left(t X_{2}\right) p \exp \left(-t X_{1}\right)\right|_{t=0}(p \in G) .
$$

Then

$$
\left(X^{*}\right)_{p}=\left(d L_{p}\right)\left(\operatorname{Ad}(p)^{-1} X_{2}-X_{1}\right)
$$

holds. If $X_{2}=0$, then $X^{*}$ is a left invariant vector field. Denote by $\nabla$ the Levi-Civita connection on $G$. By Koszul's formula, we have the following.

Lemma 3. Let $g \in G, X=\left(X_{2}, X_{1}\right), Y=\left(Y_{2}, Y_{1}\right) \in \mathfrak{g} \times \mathfrak{g}$. Then we have

$$
\left(\nabla_{X^{*}} Y^{*}\right)_{g}=-\frac{1}{2} d L_{g}\left[\operatorname{Ad}(g)^{-1} X_{2}-X_{1}, \operatorname{Ad}(g)^{-1} Y_{2}+Y_{1}\right]
$$

ProOF. By Koszul's formula, we have

$$
2\left\langle\nabla_{X^{*}} Y^{*}, Z\right\rangle=X^{*}\left\langle Y^{*}, Z\right\rangle+Y^{*}\left\langle Z, X^{*}\right\rangle-Z\left\langle X^{*}, Y^{*}\right\rangle
$$




$$
+\left\langle\left[X^{*}, Y^{*}\right], Z\right\rangle-\left\langle\left[Y^{*}, Z\right], X^{*}\right\rangle+\left\langle\left[Z, X^{*}\right], Y^{*}\right\rangle
$$

for any $X=\left(X_{2}, X_{1}\right), Y=\left(Y_{2}, Y_{1}\right) \in \mathfrak{g} \times \mathfrak{g}, Z \in \mathfrak{g}$. Computing the right side of the above equation at $e$, we have

$$
2\left(\left\langle\nabla_{X^{*}} Y^{*}, Z\right\rangle\right)_{e}=\left\langle-\operatorname{ad}\left(X_{2}-X_{1}\right)\left(Y_{2}+Y_{1}\right), Z\right\rangle
$$

for all $Z \in \mathfrak{g}$. Hence we obtain

$$
\left(\nabla_{X^{*}} Y^{*}\right)_{e}=-\frac{1}{2}\left[X_{2}-X_{1}, Y_{2}+Y_{1}\right] .
$$

Since $d L_{g}$ is an isometry, we have

$$
\left(\nabla_{X^{*}} Y^{*}\right)_{g}=d L_{g}\left(\nabla_{d L_{g}^{-1} X^{*}} d L_{g}^{-1} Y^{*}\right)_{e} .
$$

Further, we have

$$
\begin{aligned}
\left(d L_{g}^{-1} X^{*}\right)_{h}=d L_{g}^{-1}\left(X^{*}\right)_{g h} & =d L_{g}^{-1} d L_{g h}\left(\operatorname{Ad}(g h)^{-1} X_{2}-X_{1}\right) \\
& =d L_{h}\left(\operatorname{Ad}(h)^{-1} \operatorname{Ad}(g)^{-1} X_{2}-X_{1}\right) \\
& =\left(\operatorname{Ad}(g)^{-1} X_{2}, X_{1}\right)_{h}^{*} \quad(h \in G) .
\end{aligned}
$$

Thus,

$$
d L_{g}^{-1} X^{*}=\left(\operatorname{Ad}(g)^{-1} X_{2}, X_{1}\right)^{*}
$$

holds. Summarizing the above, we obtain

$$
\left(\nabla_{X^{*}} Y^{*}\right)_{g}=-\frac{1}{2} d L_{g}\left[\operatorname{Ad}(g)^{-1} X_{2}-X_{1}, \operatorname{Ad}(g)^{-1} Y_{2}+Y_{1}\right]
$$

For $H \in \mathfrak{a}$, we denote the second fundamental form of the orbit $K_{2} g K_{1} \subset G$ by $B_{H}$. By Lemma 3, we can express $B_{H}$ for $H \in \mathfrak{a}$.

THEOREM 3. For $H \in \mathfrak{a}$, we set $g=\exp (H)$ and

$$
\begin{aligned}
V_{1} & =\sum_{\lambda \in \Sigma^{+} \backslash \Sigma_{H}} \mathfrak{m}_{\lambda} \oplus \sum_{\alpha \in W^{+} \backslash W_{H}} V_{\alpha}^{\perp}\left(\mathfrak{m}_{1} \cap \mathfrak{k}_{2}\right), \\
V_{2} & =\sum_{\lambda \in \Sigma^{+}} \mathfrak{k}_{\lambda} \oplus \sum_{\alpha \in W^{+}} V_{\alpha}^{\perp}\left(\mathfrak{k}_{1} \cap \mathfrak{m}_{2}\right) .
\end{aligned}
$$

Then we have the following:

1. For $X \in \mathfrak{k}_{0}, B_{H}\left(d L_{g}(X), Y\right)=0 \quad$ where $Y \in T_{g}\left(K_{2} g K_{1}\right)$.

2. For $X \in V\left(\mathfrak{k}_{1} \cap \mathfrak{m}_{2}\right)$,

$$
d L_{g}^{-1} B_{H}\left(d L_{g}(X), d L_{g}(Y)\right)= \begin{cases}0 & \left(Y \in \mathfrak{k}_{1} \oplus V\left(\mathfrak{m}_{1} \cap \mathfrak{k}_{2}\right)\right) \\ -\frac{1}{2}[X, Y]^{\perp} & \left(Y \in V_{1}\right) .\end{cases}
$$


3. For $X \in V\left(\mathfrak{m}_{1} \cap \mathfrak{k}_{2}\right)$,

$$
d L_{g}^{-1} B_{H}\left(d L_{g}(X), d L_{g}(Y)\right)= \begin{cases}0 & \left(Y \in V\left(\mathfrak{m}_{1} \cap \mathfrak{k}_{2}\right) \oplus V_{1}\right) \\ \frac{1}{2}[X, Y]^{\perp} & \left(Y \in V_{2}\right) .\end{cases}
$$

4. For $S_{\lambda, i}\left(\lambda \in \Sigma^{+}, 1 \leq i \leq m(\lambda)\right)$,

$$
d L_{g}^{-1} B_{H}\left(d L_{g}\left(S_{\lambda, i}\right), d L_{g}(Y)\right)= \begin{cases}0 & \left(Y \in V_{2}\right) \\ -\frac{1}{2}\left[S_{\lambda, i}, Y\right]^{\perp} & \left(Y \in V_{1}\right) .\end{cases}
$$

5. For $X_{\alpha, i}\left(\alpha \in W^{+}, 1 \leq i \leq n(\alpha)\right)$,

$$
d L_{g}^{-1} B_{H}\left(d L_{g}\left(X_{\alpha, i}\right), d L_{g}(Y)\right)= \begin{cases}0 & \left(Y \in V_{2}\right) \\ -\frac{1}{2}\left[X_{\alpha, i}, Y\right]^{\perp} & \left(Y \in V_{1}\right) .\end{cases}
$$

6. For $T_{\lambda, i}\left(\lambda \in \Sigma^{+} \backslash \Sigma_{H}, 1 \leq i \leq m(\lambda)\right)$,

- $d L_{g}^{-1} B_{H}\left(d L_{g}\left(T_{\lambda, i}\right), d L_{g}\left(T_{\mu, j}\right)\right)=\cot \langle\mu, H\rangle\left[T_{\lambda, i}, S_{\mu, j}\right]^{\perp}$ where $\mu \in \Sigma^{+} \backslash \Sigma_{H}, 1 \leq j \leq m(\mu)$.

- $d L_{g}^{-1} B_{H}\left(d L_{g}\left(T_{\lambda, i}\right), d L_{g}\left(Y_{\beta, j}\right)\right)=-\tan \langle\beta, H\rangle\left[T_{\lambda, i}, X_{\beta, j}\right]^{\perp}$ where $\beta \in W^{+} \backslash W_{H}, 1 \leq j \leq n(\beta)$.

7. For $Y_{\alpha, i}\left(\alpha \in W^{+} \backslash W_{H}, 1 \leq i \leq n(\alpha)\right)$,

$$
d L_{g}^{-1} B_{H}\left(d L_{g}\left(Y_{\alpha, i}\right), d L_{g}\left(Y_{\beta, j}\right)\right)=-\tan \langle\beta, H\rangle\left[Y_{\alpha, i}, X_{\beta, j}\right]^{\perp}
$$

where $\left.\beta \in W^{+} \backslash W_{H}, 1 \leq j \leq n(\beta)\right)$.

Here, $X^{\perp}$ is the normal component, i.e. the $\left(\left(\operatorname{Ad}(g)^{-1} \mathfrak{m}_{2}\right) \cap \mathfrak{m}_{1}\right)$-component, of a tangent vector $X \in \mathfrak{g}$.

ProOF. By a simple calculation, we have the following:

- For $X \in \mathfrak{k}_{0}, d L_{g}(X)=(X, 0)_{g}^{*}$.

- For $X \in V\left(\mathfrak{k}_{1} \cap \mathfrak{m}_{2}\right), d L_{g}(X)=(0,-X)_{g}^{*}$.

- For $X \in V\left(\mathfrak{m}_{1} \cap \mathfrak{k}_{2}\right), d L_{g}(X)=(X, 0)_{g}^{*}$.

- For $S_{\lambda, i}\left(\lambda \in \Sigma^{+}, 1 \leq i \leq m(\lambda)\right), d L_{g}\left(S_{\lambda, i}\right)=\left(0,-S_{\lambda, i}\right)_{g}^{*}$.

- For $T_{\lambda, i}\left(\lambda \in \Sigma^{+} \backslash \Sigma_{H}, 1 \leq i \leq m(\lambda)\right)$,

$$
d L_{g}\left(T_{\lambda, i}\right)=\left(-\frac{S_{\lambda, i}}{\sin \langle\lambda, H\rangle},-\cot \langle\lambda, H\rangle S_{\lambda, i}\right)_{g}^{*} .
$$

- For $X_{\alpha, i}\left(\alpha \in W^{+}, 1 \leq i \leq n(\alpha)\right), d L_{g}\left(X_{\alpha, i}\right)=\left(0,-X_{\alpha, i}\right)_{g}^{*}$. 
- For $Y_{\alpha, i}\left(\alpha \in W^{+} \backslash W_{H}, 1 \leq i \leq n(\alpha)\right)$,

$$
d L_{g}\left(Y_{\alpha, i}\right)=\left(\frac{Y_{\alpha, i}}{\cos \langle\alpha, H\rangle}, \tan \langle\alpha, H\rangle X_{\alpha, i}\right)_{g}^{*} .
$$

Then, applying Lemma 3, we have the consequence. We show only 3, since other cases showed by similar calculation. If $Y \in V\left(\mathfrak{m}_{1} \cap \mathfrak{k}_{2}\right)$, then we have

$$
d L_{g}^{-1} B_{H}\left(d L_{g}(X), d L_{g}(Y)\right)=-\frac{1}{2}[X, Y]^{\perp}=0,
$$

since $[X, Y] \in \mathfrak{k}_{1} \cap \mathfrak{k}_{2}$. If $Y=T_{\lambda, i}\left(\lambda \in \Sigma^{+} \backslash \Sigma_{H}, 1 \leq i \leq m(\lambda)\right)$, then we have

$$
\begin{aligned}
d L_{g}^{-1} B_{H}\left(d L_{g}(X), d L_{g}\left(T_{\lambda, i}\right)\right) & =-\frac{1}{2}\left[X, T_{\lambda, i}-2 \cot \langle\lambda, H\rangle S_{\lambda, i}\right]^{\perp} \\
& =\left[X, \cot \langle\lambda, H\rangle S_{\lambda, i}\right]^{\perp} .
\end{aligned}
$$

Since $\left[X, S_{\lambda, i}\right] \in V_{\lambda}^{\perp}\left(\mathfrak{m}_{1} \cap \mathfrak{k}_{2}\right)$, we consider the following three cases. When $\lambda \notin W, V_{\lambda}^{\perp}\left(\mathfrak{m}_{1} \cap\right.$ $\left.\mathfrak{k}_{2}\right)=\{0\}$. Thus $\left[X, S_{\lambda, i}\right]=0$. When $\lambda \in W \backslash W_{H}$, then $\left[X, \cot \langle\lambda, H\rangle S_{\lambda, i}\right]$ is a tangent vector. Thus $\left[X, \cot \langle\lambda, H\rangle S_{\lambda, i}\right]^{\perp}=0$. When $\lambda \in W_{H}, \cot \langle\lambda, H\rangle=0$, since $\langle\lambda, H\rangle \in(\pi / 2)+(\pi \mathbf{Z})$. Thus $\left[X, \cot \langle\lambda, H\rangle S_{\lambda, i}\right]^{\perp}=0$. If $Y=Y_{\alpha, j}\left(\alpha \in W^{+} \backslash W_{H}, 1 \leq j \leq n(\lambda)\right)$, then we have

$$
d L_{g}^{-1} B_{H}\left(d L_{g}(X), d L_{g}\left(Y_{\alpha, j}\right)\right)=\left[X, \tan \langle\alpha, H\rangle X_{\alpha, j}\right]^{\perp} .
$$

Since $\left[X, X_{\alpha, j}\right] \in \mathfrak{m}_{\alpha}$, we consider the following three cases. When $\alpha \notin \Sigma, \mathfrak{m}_{\alpha}=\{0\}$. Thus $\left[X, X_{\alpha, j}\right]=0$. When $\alpha \in \Sigma \backslash \Sigma_{H}$, then $\left[X, \tan \langle\alpha, H\rangle X_{\alpha, j}\right]$ is a tangent vector. Thus $\left[X, \tan \langle\alpha, H\rangle X_{\alpha, j}\right]^{\perp}=0$. When $\alpha \in \Sigma_{H}, \tan \langle\alpha, H\rangle=0$, since $\langle\alpha, H\rangle \in(\pi \mathbf{Z})$. Thus $\left[X, \tan \langle\alpha, H\rangle X_{\alpha, j}\right]^{\perp}=0$. If $Y \in V_{2} \subset \mathfrak{k}_{1}$, then we have

$$
d L_{g}^{-1} B_{H}\left(d L_{g}(X), d L_{g}(Y)\right)=-\frac{1}{2}[X,-Y]^{\perp}=\frac{1}{2}[X, Y]^{\perp} .
$$

By the above arguments, we have 3 .

We denote the mean curvature vector of the orbit $K_{2} g K_{1}$ at $g$ by $m_{H}$. By Theorem 3, we can see that the following corollary.

COROllary 2. For $H \in \mathfrak{a}$,

$$
d L_{g}^{-1} m_{H}=-\sum_{\lambda \in \Sigma^{+} \backslash \Sigma_{H}} m(\lambda) \cot \langle\lambda, H\rangle \lambda+\sum_{\alpha \in W^{+} \backslash W_{H}} n(\alpha) \tan \langle\alpha, H\rangle \alpha .
$$

Moreover, $d L_{g}^{-1} m_{H}=d L_{g}^{-1} m_{H}^{1}$ holds. Hence, an orbit $K_{2} g K_{1} \subset G$ is minimal if and only if $K_{2} \pi_{1}(g) \subset M_{1}$ is minimal.

Next, we consider austere orbits of the $\left(K_{2} \times K_{1}\right)$-action on $G$. By using $(\tilde{\Sigma}, \Sigma, W)$, Ikawa gave a necessary and sufficient condition for an orbit of the $K_{2}$-action to be an austere submanifold. Similarly, in the $\left(K_{2} \times K_{1}\right)$-action, we also have a necessary and sufficient 
condition for an orbit to be an austere submanifold. We investigate the set of eigenvalues of the shape operator $A^{d L_{g} \xi}$ of $K_{1} g K_{2} \subset G$ for each normal vector $d L_{g} \xi \in T_{g}^{\perp} K_{2} g K_{1} \cong$ $d L_{g}\left(\left(\operatorname{Ad}(g)^{-1} \mathfrak{m}_{2}\right) \cap \mathfrak{m}_{1}\right)$. For each $g \in G$, we denote the isotropy subgroup of the $\left(K_{2} \times K_{1}\right)$ action on $G$ at $g$ by $\left(K_{2} \times K_{1}\right)_{g}$. Notice that $\left(K_{2} \times K_{1}\right)_{g}$ is isomorphic to the isotropy subgroup $\left(K_{1}\right)_{\pi_{2}(g)}$ of the $K_{1}$-action at $\pi_{2}(g)$. The isotropy subgroup $\left(K_{2} \times K_{1}\right)_{g}$ acts on the normal space $T_{g}^{\perp}\left(K_{2} g K_{1}\right)$ by the differential of the $\left(K_{2} \times K_{1}\right)$-action. Then we have

$$
d\left(k_{2}, k_{1}\right)_{g}\left(d L_{g}(\xi)\right)=\left.\frac{d}{d t} k_{2} g \exp (t \xi) k_{1}^{-1}\right|_{t=0}=d L_{g}\left(\operatorname{Ad}\left(k_{1}\right) \xi\right) .
$$

Therefore, the representation of $\left(K_{2} \times K_{1}\right) g$ is equivalent to the adjoint representation of $\left(K_{1}\right)_{\pi_{2}(g)}$ on $\left(\operatorname{Ad}(g)^{-1} \mathfrak{m}_{2}\right) \cap \mathfrak{m}_{1}$. Since $\operatorname{Lie}\left(\left(K_{1}\right)_{\pi_{2}(g)}\right)=\mathfrak{k}_{1} \cap\left(\operatorname{Ad}(g)^{-1} \mathfrak{k}_{2}\right)$, the Lie algebra $\operatorname{Lie}\left(\left(K_{1}\right)_{\pi_{2}(g)}\right) \oplus\left(\left(\operatorname{Ad}(g)^{-1} \mathfrak{m}_{2}\right) \cap \mathfrak{m}_{1}\right)$ is an orthogonal symmetric Lie algebra with respect to $\theta_{1}$. Moreover, when $g \in \exp (\mathfrak{a}), \mathfrak{a}$ is a maximal abelian subspace of $\left(\left(\operatorname{Ad}(g)^{-1} \mathfrak{m}_{2}\right) \cap \mathfrak{m}_{1}\right)$. Thus, $\mathfrak{a}$ is a section of the representation of $\left(K_{1}\right)_{\pi_{2}(g)}$ on $\left(\operatorname{Ad}(g)^{-1} \mathfrak{m}_{2}\right) \cap \mathfrak{m}_{1}$. Therefore, we have

$$
\bigcup_{\left(k_{2}, k_{1}\right) \in\left(K_{2} \times K_{1}\right)_{g}} d\left(k_{2}, k_{1}\right)_{g} d L_{g} \mathfrak{a}=T_{g}^{\perp} K_{2} g K_{1} .
$$

Thus, without loss of generality we can assume $\xi \in \mathfrak{a}$. Hence, by Theorem 3 we have

$$
\begin{aligned}
& A^{d L_{g} \xi}\left(d L_{g}\left(S_{\lambda, i}\right), d L_{g}\left(T_{\lambda, i}\right)\right) \\
& =\left(d L_{g}\left(S_{\lambda, i}\right), d L_{g}\left(T_{\lambda, i}\right)\right)\left[\begin{array}{cc}
0 & -(1 / 2)\langle\lambda, \xi\rangle \\
-(1 / 2)\langle\lambda, \xi\rangle & -\cot \langle\lambda, H\rangle\langle\lambda, \xi\rangle
\end{array}\right] \\
& \left(\lambda \in \Sigma^{+} \backslash \Sigma_{H}, 1 \leq i \leq m(\lambda)\right), \\
& A^{d L_{g} \xi}\left(d L_{g}\left(X_{\alpha, j}\right), d L_{g}\left(Y_{\alpha, j}\right)\right) \\
& =\left(d L_{g}\left(X_{\alpha, j}\right), d L_{g}\left(Y_{\alpha, j}\right)\right)\left[\begin{array}{cc}
0 & -(1 / 2)\langle\alpha, \xi\rangle \\
-(1 / 2)\langle\alpha, \xi\rangle & \tan \langle\alpha, H\rangle\langle\alpha, \xi\rangle
\end{array}\right] \\
& \left(\alpha \in W^{+} \backslash W_{H}, 1 \leq j \leq n(\alpha)\right),
\end{aligned}
$$

and for $X \in \mathfrak{k}_{0} \oplus V\left(\mathfrak{k}_{1} \cap \mathfrak{m}_{2}\right) \oplus V\left(\mathfrak{m}_{1} \cap \mathfrak{k}_{2}\right) \oplus \sum_{\lambda \in \Sigma_{H}^{+}} \mathfrak{k}_{\lambda} \oplus \sum_{\alpha \in W_{H}^{+}} V_{\alpha}^{\perp}\left(\mathfrak{k}_{1} \cap \mathfrak{m}_{2}\right)$,

$$
A^{d L_{g} \xi} d L_{g}(X)=0 \text {. }
$$

Therefore, the set of eigenvalues of $A^{d L_{g} \xi}$ is given by

$$
\begin{aligned}
& \left.\left\{-\frac{\cos \langle\lambda, H\rangle \pm 1}{2 \sin \langle\lambda, H\rangle}\langle\lambda, \xi\rangle \text { (multiplicity }=m(\lambda)\right) \mid \lambda \in \Sigma^{+} \backslash \Sigma_{H}\right\} \\
\cup & \left.\left\{\frac{\sin \langle\alpha, H\rangle \pm 1}{2 \cos \langle\alpha, H\rangle}\langle\alpha, \xi\rangle \text { (multiplicity }=n(\alpha)\right) \mid \alpha \in W^{+} \backslash W_{H}\right\} \\
\cup & \{0 \text { (multiplicity }=l)\}
\end{aligned}
$$


where $l=\operatorname{dim}\left(\mathfrak{k}_{0} \oplus \sum_{\lambda \in \Sigma_{H}} \mathfrak{k}_{\lambda} \oplus V\left(\mathfrak{k}_{1} \cap \mathfrak{m}_{2}\right) \oplus \sum_{\alpha \in W_{H}} V_{\alpha}^{\perp}\left(\mathfrak{k}_{1} \cap \mathfrak{m}_{2}\right) \oplus V\left(\mathfrak{m}_{1} \cap \mathfrak{k}_{2}\right)\right)$.

PROPOSITION 4 ([6] p.459). Let $E$ be a finite subset of a finite dimensional vector space $\mathfrak{a}$ with an inner product $\langle$,$\rangle . Then, (i) and (ii) are equivalent.$

(i) For any $\xi \in \mathfrak{a}$, the set $\{\langle a, \xi\rangle \mid a \in E\}$ with multiplicity is invariant under the multiplication by -1 .

(ii) The set $E$ is invariant under the multiplication by -1 .

Thus, we have the following corollary.

Corollary 3. Let $g=\exp (H)(H \in \mathfrak{a})$. Then the orbit $K_{2} g K_{1} \subset G$ is austere if and only if the finite subset of $\mathfrak{a}$ defined by

$$
\begin{aligned}
& \left.\left\{-\frac{\cos \langle\lambda, H\rangle \pm 1}{2 \sin \langle\lambda, H\rangle} \lambda \text { (multiplicity }=m(\lambda)\right) \mid \lambda \in \Sigma^{+} \backslash \Sigma_{H}\right\} \\
& \left.\cup\left\{\frac{\sin \langle\alpha, H\rangle \pm 1}{2 \cos \langle\alpha, H\rangle} \alpha \text { (multiplicity }=n(\alpha)\right) \mid \alpha \in W^{+} \backslash W_{H}\right\}
\end{aligned}
$$

is invariant under the multiplication by -1 .

It is easy to prove that the following proposition.

Proposition 5. For each $H \in \mathfrak{a}$,

$$
\begin{gathered}
\left.E=\{-\lambda \cot \langle\lambda, H\rangle \text { (multiplicity }=m(\lambda)) \mid \lambda \in \Sigma^{+} \backslash \Sigma_{H}\right\} \\
\left.\cup\{\alpha \tan \langle\alpha, H\rangle \text { (multiplicity }=n(\alpha)) \mid \alpha \in W^{+} \backslash W_{H}\right\}
\end{gathered}
$$

is invariant under the multiplication by -1 with multiplicities if and only if

$$
\begin{aligned}
E^{\prime}= & \left.\left\{-\frac{\cos \langle\lambda, H\rangle \pm 1}{2 \sin \langle\lambda, H\rangle} \lambda \text { (multiplicity }=m(\lambda)\right) \mid \lambda \in \Sigma^{+} \backslash \Sigma_{H}\right\} \\
& \left.\cup\left\{\frac{\sin \langle\alpha, H\rangle \pm 1}{2 \cos \langle\alpha, H\rangle} \alpha \text { (multiplicity }=n(\alpha)\right) \mid \alpha \in W^{+} \backslash W_{H}\right\}
\end{aligned}
$$

is invariant under the multiplication by -1 with multiplicities.

Proof. The equation $E=-E$ holds if and only if (i) and (ii) hold, where

(i) $\langle\lambda, H\rangle \in(\pi / 4) \mathbf{Z} \quad\left(\lambda \in \tilde{\Sigma}^{+} \backslash \tilde{\Sigma}_{H}\right)$,

(ii) if $\langle\lambda, H\rangle \in(\pi / 4)+(\pi / 2) \mathbf{Z}$, then $m(\lambda)=n(\lambda)$.

When $E=-E$ holds, for each $\lambda \in \tilde{\Sigma}^{+} \backslash \tilde{\Sigma}_{H}$, if $\langle\lambda, H\rangle \in(\pi / 2) \mathbf{Z}$, then it holds either one of the following:

- $\lambda \in \Sigma_{H}$ and

$$
\frac{\sin \langle\lambda, H\rangle+1}{2 \cos \langle\lambda, H\rangle}=-\frac{\sin \langle\lambda, H\rangle-1}{2 \cos \langle\lambda, H\rangle}
$$


- $\lambda \in W_{H}$ and

$$
-\frac{\cos \langle\lambda, H\rangle+1}{2 \sin \langle\lambda, H\rangle}=\frac{\cos \langle\lambda, H\rangle-1}{2 \sin \langle\lambda, H\rangle} .
$$

Further, if $\langle\lambda, H\rangle \in(\pi / 4)+(\pi / 2) \mathbf{Z}$, then it holds either one of the following:

- $m(\lambda)=n(\lambda)$ and

$$
\frac{\cos \langle\lambda, H\rangle+1}{2 \sin \langle\lambda, H\rangle}=\frac{\sin \langle\lambda, H\rangle+1}{2 \cos \langle\lambda, H\rangle}, \quad \text { and } \quad \frac{\cos \langle\lambda, H\rangle-1}{2 \sin \langle\lambda, H\rangle}=\frac{\sin \langle\lambda, H\rangle-1}{2 \cos \langle\lambda, H\rangle} .
$$

- $m(\lambda)=n(\lambda)$ and

$$
\frac{\cos \langle\lambda, H\rangle+1}{2 \sin \langle\lambda, H\rangle}=\frac{\sin \langle\lambda, H\rangle-1}{2 \cos \langle\lambda, H\rangle}, \quad \text { and } \quad \frac{\cos \langle\lambda, H\rangle-1}{2 \sin \langle\lambda, H\rangle}=\frac{\sin \langle\lambda, H\rangle+1}{2 \cos \langle\lambda, H\rangle} .
$$

This implies that $E^{\prime}=-E^{\prime}$. The converse is shown by the same way.

Corollary 4. Let $g=\exp (H)(H \in \mathfrak{a})$. The orbit $K_{2} g K_{1} \subset G$ is austere if and only if $K_{2} \pi_{1}(g) \subset M_{1}$ is austere.

REMARK 1. There is no correspondence in totally geodesic orbits. For example, when $\theta_{1}$ and $\theta_{2}$ cannot be transformed each other by an inner automorphism of $\mathfrak{g}, K_{2} e K_{1} \subset G$ is not totally geodesic, but $K_{2} \pi_{1}(e) \subset M_{1}$ is totally geodesic (see (4) and (5) in Theorem 3).

\section{Main Theorem}

In the previous section, we saw a correspondence of austereness of orbits of the $\left(K_{2} \times\right.$ $K_{1}$ )-action and the $K_{2}$-action. In this section, we consider weakly reflective orbits of the $\left(K_{2} \times K_{1}\right)$-action, the $K_{2}$-action and the $K_{1}$-action, and give two sufficient conditions for an orbit to be weakly reflective. The first sufficient condition is the following:

THEOREM 4. Assume $K_{1}$ and $K_{2}$ are connected. Let $g=\exp (H)(H \in \mathfrak{a})$. If $\langle\lambda, H\rangle \in(\pi / 2) \mathbf{Z}$ for any $\lambda \in \tilde{\Sigma}$, that is, $H \in \Gamma$, then the orbit $K_{2} g K_{1} \subset G$ is weakly reflective.

Proof. We set $\sigma=L_{g} \theta_{1} L_{g}^{-1}$. Then $\sigma$ satisfies the following conditions:

1. $\sigma(g)=g$,

2. $\sigma\left(K_{2} g K_{1}\right)=K_{2} g K_{1}$,

3. $d \sigma(\xi)=-\xi \quad\left(\xi \in T_{g}^{\perp}\left(K_{2} g K_{1}\right)\right)$.

Clearly, $\sigma(g)=g$ holds. By Lemma 2, we have $\operatorname{Ad}\left(g^{2}\right) \mathfrak{k}_{2}=\mathfrak{k}_{2}$. Since $K_{2}$ is connected, we have $g^{2} K_{2} g^{-2}=K_{2}$. In addition, since $\theta_{1} \theta_{2}=\theta_{2} \theta_{1}$, we have $\theta_{1} \mathfrak{k}_{2}=\mathfrak{k}_{2}$. Thus, we also have $\theta_{1}\left(K_{2}\right)=K_{2}$. Therefore, for $\left(k_{2}, k_{1}\right) \in K_{2} \times K_{1}$,

$$
\sigma\left(k_{2} g k_{1}^{-1}\right)=\left(g^{2} \theta_{1}\left(k_{2}\right) g^{-2}\right) g k_{1}^{-1} \in K_{2} g K_{1} .
$$


Hence, $\sigma\left(K_{2} g K_{1}\right)=K_{2} g K_{1}$. Since $T_{g}^{\perp}\left(K_{2} g K_{1}\right)=d L_{g}\left(\operatorname{Ad}(g)^{-1}\left(\mathfrak{m}_{2}\right) \cap \mathfrak{m}_{1}\right)$, we have

$$
d \sigma(\xi)=d L_{g} \theta_{1}\left(d L_{g}^{-1}(\xi)\right)=-d L_{g} d L_{g}^{-1}(\xi)=-\xi
$$

Therefore, $\sigma$ is a reflection of $K_{2} g K_{1}$ at $g$ with respect to each normal vector $d L_{g} \xi \in$ $T_{g}^{\perp}\left(K_{2} g K_{1}\right)$.

COROLlaRY 5. The orbit $K_{2} e K_{1} \subset G$ is weakly reflective.

REMARK 2. Under the same condition as Theorem 4, we can prove that $K_{2} \pi_{1}(g) \subset$ $M_{1}$ and $K_{1} \pi_{2}(g) \subset M_{2}$ are weakly reflective. However, Ikawa proved $K_{2} \pi_{1}(g) \subset M_{1}$ and $K_{1} \pi_{2}(g) \subset M_{2}$ are reflective. Hence $K_{2} \pi_{1}(g) \subset M_{1}$ and $K_{1} \pi_{2}(g) \subset M_{2}$ are totally geodesic, but $K_{2} g K_{1}$ is not necessarily totally geodesic. In fact, when $\theta_{1}$ and $\theta_{2}$ cannot be transformed each other by inner automorphism of $\mathfrak{g}$, then there is no totally geodesic orbit of the $\left(K_{2} \times K_{1}\right)$-action on $G$.

Let $\tilde{W}(\tilde{\Sigma}, \Sigma, W)$ be a subgroup of the affine group $\mathrm{O}(\mathfrak{a}) \ltimes \mathfrak{a}$ which is generated by

$$
\left\{\left(s_{\lambda}, \frac{2 n \pi}{\langle\lambda, \lambda\rangle} \lambda\right) \mid \lambda \in \Sigma, n \in \mathbf{Z}\right\} \cup\left\{\left(s_{\alpha}, \frac{(2 n+1) \pi}{\langle\alpha, \alpha\rangle} \alpha\right) \mid \alpha \in W, n \in \mathbf{Z}\right\} .
$$

Then, we have the following lemma.

LEMMA 4 ([4] Lemmas 4.4 and 4.21).

$$
\tilde{W}(\tilde{\Sigma}, \Sigma, W) \subset \tilde{J}
$$

Using the above lemma, we have the following lemma.

Lemma 5. Let $g=\exp (H)(H \in \mathfrak{a})$. Then, for each $\lambda \in \tilde{\Sigma}_{H}$, there exists $k_{\lambda} \in$ $N_{K_{2}}(\mathfrak{a})$, such that

1.

$$
\left(k_{\lambda}, \exp \left(-\frac{2\langle\lambda, H\rangle}{\langle\lambda, \lambda\rangle} \lambda\right) k_{\lambda}\right) \in\left(K_{2} \times K_{1}\right)_{g}
$$

2.

$$
d\left(k_{\lambda}, \exp \left(-\frac{2\langle\lambda, H\rangle}{\langle\lambda, \lambda\rangle} \lambda\right) k_{\lambda}\right)_{g}\left(d L_{g} \xi\right)=d L_{g}\left(s_{\lambda} \xi\right) \quad(\xi \in \mathfrak{a})
$$

Proof. By the definition of $\tilde{W}(\tilde{\Sigma}, \Sigma, W)$, for each $\lambda \in \tilde{\Sigma}_{H}$,

$$
\left(s_{\lambda}, 2 \frac{\langle\lambda, H\rangle}{\langle\lambda, \lambda\rangle} \lambda\right) \in \tilde{W}(\tilde{\Sigma}, \Sigma, W) .
$$

Since $\tilde{W}(\tilde{\Sigma}, \Sigma, W) \subset \tilde{J}$, there exists $k_{\lambda} \in N_{K_{2}}(\mathfrak{a})$, such that

$$
\left(\left[k_{\lambda}\right], 2 \frac{\langle\lambda, H\rangle}{\langle\lambda, \lambda\rangle} \lambda\right)=\left(s_{\lambda}, 2 \frac{\langle\lambda, H\rangle}{\langle\lambda, \lambda\rangle} \lambda\right) .
$$


By the definition of $\tilde{J}$, we have

$$
\exp \left(-2 \frac{\langle\lambda, H\rangle}{\langle\lambda, \lambda\rangle} \lambda\right) k_{\lambda} \in K_{1}
$$

For 1,

$$
\begin{aligned}
& \left(k_{\lambda}, \exp \left(-\frac{2\langle\lambda, H\rangle}{\langle\lambda, \lambda\rangle} \lambda\right) k_{\lambda}\right) g=k_{\lambda} \exp (H) k_{\lambda}^{-1} \exp \left(\frac{2\langle\lambda, H\rangle}{\langle\lambda, \lambda\rangle} \lambda\right) \\
= & \exp \left(\operatorname{Ad}\left(k_{\lambda}\right) H\right) \exp \left(\frac{2\langle\lambda, H\rangle}{\langle\lambda, \lambda\rangle} \lambda\right)=\exp \left(s_{\lambda} H+\frac{2\langle\lambda, H\rangle}{\langle\lambda, \lambda\rangle} \lambda\right)=\exp (H)=g .
\end{aligned}
$$

For 2,

$$
d\left(k_{\lambda}, \exp \left(-\frac{2\langle\lambda, H\rangle}{\langle\lambda, \lambda\rangle} \lambda\right) k_{\lambda}\right)_{g}\left(d L_{g} \xi\right)=\left.\frac{d}{d t} \exp \left(H+t s_{\lambda}(\xi)\right)\right|_{t=0}=d L_{g} s_{\lambda}(\xi) .
$$

Proposition 6. For any $H \in \mathfrak{a}$, if $\tilde{\Sigma}_{H}$ is nonempty, then $\tilde{\Sigma}_{H}$ is a root system of $\operatorname{Span}\left(\tilde{\Sigma}_{H}\right)$.

Proof. We set $g=\exp (H)$. We consider the orthogonal symmetric Lie algebra

$$
\left(\left(\operatorname{Ad}(g)^{-1} \mathfrak{k}_{2}\right) \cap \mathfrak{k}_{1}\right) \oplus\left(\left(\operatorname{Ad}(g)^{-1} \mathfrak{m}_{2}\right) \cap \mathfrak{m}_{1}\right) .
$$

By Lemma 2, we can decompose the Lie algebra as the following:

$$
\left(\mathfrak{k}_{0} \oplus \sum_{\lambda \in \Sigma_{H}^{+}} \mathfrak{k}_{\lambda} \oplus \sum_{\alpha \in W_{H}^{+}} V_{\alpha}^{\perp}\left(\mathfrak{k}_{1} \cap \mathfrak{m}_{2}\right)\right) \oplus\left(\mathfrak{a} \oplus \sum_{\lambda \in \Sigma_{H}^{+}} \mathfrak{m}_{\lambda} \oplus \sum_{\alpha \in W_{H}^{+}} V_{\alpha}^{\perp}\left(\mathfrak{m}_{1} \cap \mathfrak{k}_{2}\right)\right) .
$$

It is the root space decomposition of the orthogonal symmetric Lie algebra with respect to a.

REMARK 3. By Proposition 6 and Theorem 6, for any symmetric triad of $\mathfrak{a}$ and $H \in \mathfrak{a}$, if $\tilde{\Sigma}_{H}$ is nonempty, then $\tilde{\Sigma}_{H}$ is a root system of $\operatorname{Span}\left(\tilde{\Sigma}_{H}\right)$.

For each $H \in \mathfrak{a}$, denote by $W\left(\tilde{\Sigma}_{H}\right)$ the Weyl group of $\tilde{\Sigma}_{H}$. The second sufficient condition is the following:

TheOREM 5. Let $g=\exp (H)(H \in \mathfrak{a})$. If $\operatorname{Span}\left(\tilde{\Sigma}_{H}\right)=\mathfrak{a}$ and $-\mathrm{id}_{\mathfrak{a}} \in W\left(\tilde{\Sigma}_{H}\right)$, then $K_{2} g K_{1} \subset G, K_{2} \pi_{1}(g) \subset M_{1}$ and $K_{1} \pi_{2}(g) \subset M_{2}$ are weakly reflective.

PROOF. By the equation (7), it is sufficient to prove the existence of a reflection with respect to $d L_{g} \xi$ for each $\xi \in \mathfrak{a}$. Since $-\mathrm{id}_{\mathfrak{a}} \in W\left(\tilde{\Sigma}_{H}\right)$, there exist $\mu_{1}, \ldots, \mu_{l} \in \tilde{\Sigma}_{H}$ such that $s_{\mu_{1}} \cdots s_{\mu_{l}}=-\mathrm{id}_{\mathfrak{a}}$. By Lemma 5, there exists $k_{\mu_{i}} \in N_{K_{2}}(\mathfrak{a})$ for each $\mu_{i}(1 \leq i \leq l)$. We set

$$
k_{\mu_{i}}^{\prime}=\exp \left(-2 \frac{\left\langle\mu_{i}, H\right\rangle}{\left\langle\mu_{i}, \mu_{i}\right\rangle} \mu_{i}\right) k_{\mu_{i}} \in K_{1}
$$


and

$$
\sigma=\left(k_{\mu_{1}}, k_{\mu_{1}}^{\prime}\right) \cdots\left(k_{\mu_{l}}, k_{\mu_{l}}^{\prime}\right) \in\left(K_{2} \times K_{1}\right)_{g} .
$$

Then, $\sigma$ is a reflection of $K_{2} g K_{1}$ with respect to $d L_{g} \xi$ for each $\xi \in \mathfrak{a}$. Indeed,

$$
\sigma(g)=g, \quad \sigma\left(K_{2} g K_{1}\right)=K_{2} g K_{1}, \quad d \sigma\left(d L_{g}(\xi)\right)=d L_{g} s_{\mu_{1}} \cdots s_{\mu_{l}}(\xi)=-d L_{g} \xi
$$

hold. Similarly, $\sigma_{1}=k_{\mu_{1}} \cdots k_{\mu_{l}}$ is a reflection of $K_{2} \pi_{1}(g)$ at $\pi_{1}(g)$ with respect to $d L_{g} \xi$. The isometry $\sigma_{2}=k_{\mu_{1}}^{\prime} \cdots k_{\mu_{l}}^{\prime}$ is a reflection of $K_{1} \pi_{2}(g)$ at $\pi_{2}(g)$ with respect to $d R_{g} \xi$.

In [6], they mainly studied weakly reflective submanifolds in $S^{n}$ and $\mathbf{C} P^{n}$. The cohomogeneity of Hermann actions on rank one symmetric spaces must be one. Therefore, by Proposition 1, singular orbits of Hermann actions on rank one symmetric spaces are weakly reflective. However, when the cohomogeneity of Hermann action is two or greater, applying Theorems 5 and 4 , we have new examples of weakly reflective submanifolds in compact symmetric spaces. We assume that $\left(G, K_{1}, K_{2}\right)$ satisfies one of the following conditions (A), (B) or (C).

(A) $G$ is simple and $\theta_{1}$ and $\theta_{2}$ can not transform each other by an inner automorphism of $\mathfrak{g}$.

(B) There exist a compact connected simple Lie group $U$ and a symmetric subgroup $\bar{K}$ of $U$ such that

$$
G=U \times U, \quad K_{1}=\Delta G=\{(u, u) \mid u \in U\}, \quad K_{2}=\bar{K} \times \bar{K} .
$$

(C) There exist a compact connected simple Lie group $U$ and an involutive outer automorphism $\sigma$ such that

$$
\begin{gathered}
G=U \times U, \quad K_{1}=\Delta G=\{(u, u) \mid u \in U\}, \\
K_{2}=\left\{\left(u_{1}, u_{2}\right) \mid\left(\sigma\left(u_{2}\right), \sigma\left(u_{1}\right)\right)=\left(u_{1}, u_{2}\right)\right\} .
\end{gathered}
$$

Ikawa proved the following theorem.

THEOREM 6 ([5]). Let $\left(G, K_{1}, K_{2}\right)$ be a compact symmetric triad which satisfies one of the conditions (A), (B) or (C). Then the triple $(\tilde{\Sigma}, \Sigma, W)$ defined as above is a symmetric triad with multiplicities. Conversely every symmetric triad is obtained in this way.

It is known the following proposition.

Proposition 7 ([10]). Let $\Sigma$ be an irreducible root system of $\mathfrak{a}$. Then $-\mathrm{id}_{\mathfrak{a}} \notin W(\Sigma)$ if and only if $\Sigma \cong \mathrm{A}_{r}, \mathrm{D}_{2 r+1}, \mathrm{E}_{6}(r \geq 2)$.

Let $\Pi=\left\{\lambda_{1}, \ldots, \lambda_{r}\right\}$ be a fundamental system of $\Sigma$, and set $W_{0}=\{\tilde{\alpha}\}$. We define $H_{i} \in \mathfrak{a}$ by the following equations:

$$
\left\langle H_{i}, \lambda_{j}\right\rangle=0(i \neq j),\left\langle H_{i}, \tilde{\alpha}\right\rangle=\pi / 2 .
$$

Then, $\left\{H_{1}, \ldots, H_{r}\right\}$ is a basis of $\mathfrak{a}$. We have the following lemma.

LEMMA 6. $\operatorname{Span}\left(\tilde{\Sigma}_{H}\right)=\mathfrak{a}$ if and only if $H=0, H_{1}, \ldots, H_{r}$ for $H \in \bar{P}_{0}$. 
Proof. Let $H \in \mathfrak{a}$. By definition of $\tilde{\Sigma}_{H}$, we have

$$
\left(s_{\mu_{i}}, \frac{2\langle\lambda, H\rangle}{\langle\lambda, \lambda\rangle} \lambda\right) \in \tilde{W}(\tilde{\Sigma}, \Sigma, W), \quad\left(s_{\mu_{i}}, \frac{2\langle\lambda, H\rangle}{\langle\lambda, \lambda\rangle} \lambda\right) H=H
$$

for each $\lambda \in \tilde{\Sigma}_{H}$. By Proposition 2, we have $s_{\lambda} m_{H}=m_{H}$ for $\lambda \in \tilde{\Sigma}_{H}$. Thus, if $\operatorname{Span}\left(\tilde{\Sigma}_{H}\right)=$ $\mathfrak{a}$, then $m_{H}=0$. On the other hand, for $H \in \bar{P}_{0}$, there exists the nonempty subset $\Delta \subset \Pi \cup$ $\{\tilde{\alpha}\}$ such that $H \in P_{0}^{\Delta}$. By Lemma 2.25 in [4], $\Sigma_{H}$ and $W_{H}$ does not depend on $H$, but only $\Delta$. Thus, when $\operatorname{Span}\left(\tilde{\Sigma}_{H}\right)=\mathfrak{a}$, each point in $P_{0}^{\Delta}$ is a minimal point. Therefore, by Theorem 1 , if $\operatorname{Span}\left(\tilde{\Sigma}_{H}\right)=\mathfrak{a}$, then $P_{0}^{\Delta}=\{H\}$. This implies that $H$ is a vertex of $\bar{P}_{0}$. Therefore, $H=0, H_{1}, \ldots, H_{r}$. Conversely, when $H=0, H_{1}, \ldots, H_{r}$, we have $\operatorname{Span}\left(\tilde{\Sigma}_{H}\right)=\mathfrak{a}$.

For each symmetric triad of $\mathfrak{a}$, austere points are classified in [4]. Using the classification, we investigate $\tilde{\Sigma}_{H_{i}}(1 \leq i \leq r)$ for each type of symmetric triads.

In order to state our results below, we shall follow the notations of irreducible root systems and the set of positive roots in [1]. For instance,

$$
\begin{aligned}
\mathrm{B}_{r}^{+} & =\left\{e_{i} \pm e_{j} \mid 1 \leq i<j \leq r\right\} \cup\left\{e_{i} \mid 1 \leq i \leq r\right\}, \\
\mathrm{C}_{r}^{+} & =\left\{e_{i} \pm e_{j} \mid 1 \leq i<j \leq r\right\} \cup\left\{2 e_{i} \mid 1 \leq i \leq r\right\}, \\
\mathrm{D}_{r}^{+} & =\left\{e_{i} \pm e_{j} \mid 1 \leq i<j \leq r\right\}, \\
\mathrm{BC}_{r}^{+} & =\left\{e_{i} \pm e_{j} \mid 1 \leq i<j \leq r\right\} \cup\left\{e_{i} \mid 1 \leq i \leq r\right\} \cup\left\{2 e_{i} \mid 1 \leq i \leq r\right\} .
\end{aligned}
$$

For the set of positive roots above, the sets of simple roots are given as follows:

$$
\begin{aligned}
& \Pi\left(\mathrm{B}_{r}^{+}\right)=\Pi\left(\mathrm{BC}_{r}^{+}\right)=\left\{\lambda_{1}=e_{1}-e_{2}, \ldots, \lambda_{r-1}=e_{r-1}-e_{r}, \lambda_{r}=e_{r}\right\}, \\
& \Pi\left(\mathrm{C}_{r}^{+}\right)=\left\{\lambda_{1}=e_{1}-e_{2}, \ldots, \lambda_{r-1}=e_{r-1}-e_{r}, \lambda_{r}=2 e_{r}\right\}, \\
& \Pi\left(\mathrm{D}_{r}^{+}\right)=\left\{\lambda_{1}=e_{1}-e_{2}, \ldots, \lambda_{r-1}=e_{r-1}-e_{r}, \lambda_{r}=e_{r-1}+e_{r}\right\} .
\end{aligned}
$$

4.1. Type $\mathrm{I}-\mathrm{B}_{r} . \quad \Sigma^{+}=\mathrm{B}_{r}^{+}, W^{+}=\left\{e_{i} \mid 1 \leq i \leq r\right\}$,

$$
\tilde{\alpha}=e_{1}=\lambda_{1}+\cdots+\lambda_{r} \text {. }
$$

(1) When $m\left( \pm e_{i}\right)=n\left( \pm e_{i}\right) \quad$ A point $H \in \overline{P_{0}}$ is austere which is not totally geodesic if and only if $H=(1 / 2) H_{r}$. Since $\operatorname{Span}\left(\tilde{\Sigma}_{H}\right) \neq \mathfrak{a}$, the point $(1 / 2) H_{r}$ does not satisfy the sufficient condition in Theorem 5.

(2) When $m\left( \pm e_{i}\right) \neq n\left( \pm e_{i}\right) \quad$ If $H \in \overline{P_{0}}$ is austere then it is totally geodesic. In this case, $H_{i}$ is a totally geodesic point for each $1 \leq i \leq r$.

A compact symmetric triad whose symmetric triad is type $\mathrm{I}-\mathrm{B}_{r}$ is one of the following:

1. $(\mathrm{SO}(r+s+t), \mathrm{SO}(r+s) \times \mathrm{SO}(t), \mathrm{SO}(r) \times \mathrm{SO}(s+t)) \quad(r<t, 1 \leq s)$, 
2. $\left(G, K_{1}, K_{2}\right)$ which satisfies condition (C) where

$$
(U, \operatorname{Fix}(\sigma))=(\operatorname{SO}(2 m+2 n+2), \operatorname{SO}(2 m+1) \times \operatorname{SO}(2 n+1))
$$

for $r=m+n, m \geq 2$.

4.2. Type $\mathrm{I}-\mathrm{C}_{r} . \quad \Sigma^{+}=\mathrm{C}_{r}^{+}, W^{+}=\mathrm{D}_{r}^{+}$,

$$
\tilde{\alpha}=e_{1}+e_{2}=\lambda_{1}+2 \lambda_{2}+\cdots 2 \lambda_{r-1}+\lambda_{r} .
$$

Then a point $H \in \overline{P_{0}}$ is austere which is not totally geodesic if and only if $H=H_{i}(2 \leq i \leq$ $r-1),(1 / 2) H_{1}$. For each $H_{i}=(\pi / 4)\left(e_{1}+\cdots+e_{i}\right) \quad(2 \leq i \leq r-1)$, we have

$$
\begin{aligned}
\Sigma_{H_{i}}^{+}= & \left\{e_{s}-e_{t} \mid 1 \leq s<t \leq i\right\} \cup\left\{e_{s} \pm e_{t} \mid i+1 \leq s<t \leq r\right\} \\
& \cup\left\{2 e_{s} \mid i+1 \leq s \leq r\right\}, \\
W_{H_{i}}^{+}= & \left\{e_{s}+e_{t} \mid 1 \leq s<t \leq i\right\} .
\end{aligned}
$$

Hence, $\tilde{\Sigma}_{H_{i}} \cong \mathrm{D}_{i} \oplus \mathrm{C}_{r-i}$. Therefore, by Proposition 7 and Theorem 5, if $i$ is even, then $K_{2} \exp \left(H_{i}\right) K_{1} \subset G, K_{2} \pi_{1}\left(\exp \left(H_{i}\right)\right) \subset M_{1}, K_{1} \pi_{2}\left(\exp \left(H_{i}\right)\right) \subset M_{2}$ are weakly reflective. When $i$ is odd, since $-\mathrm{id}_{\mathfrak{a}} \notin W(\Sigma), H_{i}$ does not satisfy the sufficient condition in Theorem 5.

A compact symmetric triad whose symmetric triad is type $\mathrm{I}^{-} \mathrm{C}_{r}$ is one of the following:

1. $(\mathrm{SO}(4 r), \mathrm{SO}(2 r) \times \mathrm{SO}(2 r), \mathrm{U}(2 r))$,

2. $(\mathrm{SU}(2 r), \mathrm{SO}(2 r), \mathrm{S}(\mathrm{U}(r) \times \mathrm{U}(r)))$,

3. $\left(E_{7}, \mathrm{SU}(8), E_{6} \cdot \mathrm{U}(1)\right) \quad(r=3)$,

4. $\left(G, K_{1}, K_{2}\right)$ which satisfies condition (C) where

$$
(U, \operatorname{Fix}(\sigma))=(\mathrm{SU}(2 r), \mathrm{SO}(2 r)) \quad(r \geq 2) \text { or }(\mathrm{SU}(2 r), \operatorname{Sp}(r)) \quad(r \geq 2) .
$$

4.3. Type $\mathrm{I}_{-} \mathrm{BC}_{r}-\mathrm{A}_{1}^{r} . \quad \Sigma^{+}=\mathrm{BC}_{r}^{+}, W^{+}=\left\{e_{i} \mid 1 \leq i \leq r\right\}$,

$$
\tilde{\alpha}=e_{1}=\lambda_{1}+\cdots+\lambda_{r} .
$$

(1) When $m\left( \pm e_{i}\right)=n\left( \pm e_{i}\right) \quad$ A point $H \in \overline{P_{0}}$ is austere which is not totally geodesic if and only if $H=(1 / 2) H_{r}$. Since $\operatorname{Span}\left(\tilde{\Sigma}_{H}\right) \neq \mathfrak{a}, H$ does not satisfy the sufficient condition in Theorem 5.

(2) When $m\left( \pm e_{i}\right) \neq n\left( \pm e_{i}\right) \quad$ If $H \in \overline{P_{0}}$ is austere then it is totally geodesic. In this case, $H_{i}$ is a totally geodesic point for each $1 \leq i \leq r$.

A compact symmetric triad whose symmetric triad is type $\mathrm{I}_{-}-\mathrm{BC}_{r}-\mathrm{A}_{1}^{r}$ is one of the following:

1. $(\mathrm{SU}(r+s+t), \mathrm{S}(\mathrm{U}(r+s) \times \mathrm{U}(t)), \mathrm{S}(\mathrm{U}(r) \times \mathrm{U}(s+t))) \quad(r<t, 1 \leq s)$,

2. $(\mathrm{Sp}(r+s+t), \mathrm{Sp}(r+s) \times \mathrm{Sp}(t), \mathrm{Sp}(r) \times \mathrm{Sp}(s+t)) \quad(r<t, 1 \leq s))$,

3. $\left(\mathrm{SO}(4 r+4), \mathrm{U}(2 r+2), \mathrm{U}^{\prime}(2 r+2)\right)$. 
Where, we set

$$
J=\left[\begin{array}{ll|ll} 
& & I_{n-1} & \\
& & & -1 \\
\hline-I_{n-1} & & &
\end{array}\right],
$$

and define $\mathrm{U}^{\prime}(n):=\left\{g \in \mathrm{SO}(2 n) \mid J g J^{-1}=g\right\}$.

4.4. Type $\mathrm{I}-\mathrm{BC}_{r}-\mathrm{B}_{r} . \quad \Sigma^{+}=\mathrm{BC}_{r}^{+}, W^{+}=\mathrm{B}_{r}^{+}$,

$$
\tilde{\alpha}=e_{1}+e_{2}=\lambda_{1}+2 \lambda_{2}+\cdots+2 \lambda_{r} .
$$

When $r=2$, if $m\left( \pm e_{1} \pm e_{2}\right)=n\left( \pm e_{1} \pm e_{2}\right)$, then $H \in \overline{P_{0}}$ is austere which is not totally geodesic if and only if $H=(1 / 2) H_{1}, H_{2}$. If $m\left( \pm e_{1} \pm e_{2}\right) \neq n\left( \pm e_{1} \pm e_{2}\right)$, then $H \in \overline{P_{0}}$ is austere which is not totally geodesic if and only if $H=H_{2}$. Since $H_{2}=(\pi / 4)\left(e_{1}+e_{2}\right)$, we have $\Sigma_{H_{2}}^{+}=\left\{e_{1}-e_{2}\right\}, W_{H_{2}}^{+}=\left\{e_{1}+e_{2}\right\}$. Thus $\tilde{\Sigma}_{H_{2}} \cong \mathrm{A}_{1}^{2}$. By Proposition 7 and Theorem 5, $K_{2} \exp \left(H_{2}\right) K_{1} \subset G, K_{2} \pi_{1}\left(\exp \left(H_{2}\right)\right) \subset M_{1}, K_{1} \pi_{2}\left(\exp \left(H_{2}\right)\right) \subset M_{2}$ are weakly reflective.

When $r \geq 3, H \in \overline{P_{0}}$ is austere which is not totally geodesic if and only if $H=$ $(1 / 2) H_{1}, H_{i} \quad(2 \leq i \leq r)$. For each $H_{i}=(\pi / 4)\left(e_{1}+\cdots+e_{i}\right) \quad(2 \leq i \leq r)$, we have $\tilde{\Sigma}_{H_{i}} \cong \mathrm{D}_{i} \oplus \mathrm{BC}_{1}^{r-i}$. Therefore, by Proposition 7 and Theorem 5, if $i$ is even, then $K_{2} \exp \left(H_{i}\right) K_{1} \subset G, K_{2} \pi_{1}\left(\exp \left(H_{i}\right)\right) \subset M_{1}, K_{1} \pi_{2}\left(\exp \left(H_{i}\right)\right) \subset M_{2}$ are weakly reflective for each $2 \leq i \leq r$. When $i$ is odd, since $-\operatorname{id}_{\mathfrak{a}} \notin W(\Sigma), H_{i}$ does not satisfy the sufficient condition in Theorem 5 for $3 \leq i \leq r$. Since $\operatorname{Span}\left(\tilde{\Sigma}_{(1 / 2) H_{1}}\right) \neq \mathfrak{a}$, the point $(1 / 2) H_{1}$ does not satisfy the sufficient condition in Theorem 5 .

A compact symmetric triad whose symmetric triad is type $\mathrm{I}^{-\mathrm{BC}_{r}-\mathrm{B}_{r}}$ is one of the following:

1. $(\mathrm{SO}(2 r+2 s), \mathrm{S}(\mathrm{O}(2 r) \times \mathrm{O}(2 s)), \mathrm{U}(r+s)) \quad(r<s)$,

2. $\left(E_{6}, \mathrm{SU}(6) \cdot \mathrm{SU}(2), \mathrm{SO}(10) \cdot \mathrm{U}(1)\right) \quad(r=2)$,

3. $\left(E_{7}, \mathrm{SO}(12) \cdot \mathrm{SU}(2), E_{6} \cdot \mathrm{U}(1)\right) \quad(r=2)$.

4.5. Type $\mathrm{I}-\mathrm{F}_{4} . \quad \Sigma^{+}=\mathrm{F}_{4}^{+}, W^{+}=\left\{\right.$short roots in $\left.\mathrm{F}_{4}\right\} \cong \mathrm{D}_{4}, \Pi=\left\{\lambda_{1}=e_{2}-\right.$ $\left.e_{3}, \lambda_{2}=e_{3}-e_{4}, \lambda_{3}=e_{4}, \lambda_{4}=(1 / 2)\left(e_{1}-e_{2}-e_{3}-e_{4}\right)\right\}, \tilde{\alpha}=e_{1}=\lambda_{1}+2 \lambda_{2}+3 \lambda_{3}+2 \lambda_{4}$. A point $H \in \overline{P_{0}}$ is austere which is not totally geodesic if and only if $H=H_{4}=(\pi / 2) e_{1}$. Then we have

$$
\begin{aligned}
\Sigma_{H_{4}} & =\left\{ \pm e_{2}, \pm e_{3}, \pm e_{4}, \pm\left(e_{2} \pm e_{3}\right), \pm\left(e_{2} \pm e_{4}\right), \pm\left(e_{3} \pm e_{4}\right)\right\} \\
W_{H_{4}} & =\left\{ \pm e_{1}, \pm\left(e_{1} \pm e_{2}\right), \pm\left(e_{1} \pm e_{3}\right), \pm\left(e_{1} \pm e_{4}\right)\right\}
\end{aligned}
$$

Hence

$$
\tilde{\Sigma}_{H_{4}}^{+} \cong \mathrm{B}_{4}^{+} .
$$

Therefore, by Proposition 7 and Theorem 5, the orbits $K_{2} \exp \left(H_{4}\right) K_{1} \subset G$, $K_{2} \pi_{1}\left(\exp \left(H_{4}\right)\right) \subset M_{1}$ and $K_{1} \pi_{2}\left(\exp \left(H_{4}\right)\right) \subset M_{2}$ are weakly reflective. 
A compact symmetric triad whose symmetric triad is type $\mathrm{I}_{-} \mathrm{F}_{4}$ is one of the following:

1. $\left(E_{6}, \mathrm{Sp}(4), \mathrm{SU}(6) \cdot \mathrm{SU}(2)\right)$,

2. $\left(E_{7}, \mathrm{SU}(8), \mathrm{SO}(12) \cdot \mathrm{SU}(2)\right)$,

3. $\left(E_{8}, \mathrm{SO}(16), E_{7} \cdot \mathrm{SU}(2)\right)$,

4. $\left(G, K_{1}, K_{2}\right)$ which satisfies condition (C) where

$$
(U, \operatorname{Fix}(\sigma))=\left(E_{6}, \operatorname{Sp}(4)\right) \operatorname{or}\left(E_{6}, F_{4}\right) .
$$

4.6. Type II-BC $r . \quad \Sigma^{+}=\mathrm{B}_{r}^{+}, W^{+}=\mathrm{BC}_{r}^{+}$,

$$
\tilde{\alpha}=2 e_{1}=2 \lambda_{1}+\cdots+2 \lambda_{r} .
$$

A point $H \in \overline{P_{0}}$ is austere which is not totally geodesic if and only if $H=H_{i}(1 \leq i \leq r)$. For $H_{i}=(\pi / 4)\left(e_{1}+\cdots+e_{i}\right)$, we have $\tilde{\Sigma}_{H_{i}}^{+} \cong \mathrm{C}_{i} \oplus \mathrm{B}_{r-i}$. Therefore, by Proposition 7 and Theorem 5, $K_{2} \exp \left(H_{i}\right) K_{1} \subset G, K_{2} \pi_{1}\left(\exp \left(H_{i}\right)\right) \subset M_{1}, K_{1} \pi_{2}\left(\exp \left(H_{i}\right)\right) \subset M_{2}$ are weakly reflective for each $1 \leq i \leq r$.

A compact symmetric triad whose symmetric triad is type $\mathrm{II}_{-} \mathrm{BC}_{r}$ is one of the following:

1. $(\mathrm{SU}(r+s), \mathrm{SO}(r+s), \mathrm{S}(\mathrm{U}(r) \times \mathrm{U}(s))) \quad(r<s)$,

2. $(\mathrm{SO}(4 r+2), \mathrm{SO}(2 r+1) \times \mathrm{SO}(2 r+1), \mathrm{U}(2 r+1))$,

3. $\left(E_{6}, \mathrm{Sp}(4), \mathrm{SO}(10) \cdot \mathrm{U}(1)\right) \quad(r=2)$.

4.7. Type III-A $r$. By Proposition 7, $-\mathrm{id}_{\mathfrak{a}} \notin W(\tilde{\Sigma})$. Moreover, for each $H \in \mathfrak{a}$, $W\left(\tilde{\Sigma}_{H}\right) \subset W(\tilde{\Sigma})$ since $\tilde{\Sigma}_{H} \subset \tilde{\Sigma}$. Hence $-\operatorname{id}_{\mathfrak{a}} \notin W\left(\tilde{\Sigma}_{H}\right)$. Thus, any austere point does not satisfy the sufficient condition in Theorem 5 .

A compact symmetric triad whose symmetric triad is type III- $\mathrm{A}_{r}$ is one of the following:

1. $(\mathrm{SU}(2 r+2), \mathrm{Sp}(r+1), \mathrm{SO}(2 r+2))$,

2. $\left(E_{6}, \operatorname{Sp}(4), F_{4}\right)(r=2)$,

3. $(U \times U, \Delta(U \times U), \bar{K} \times \bar{K})$ where $(U, \bar{K})$ is a compact symmetric pair whose root system is type $\mathrm{A}_{r} \quad$ (condition (B)).

4.8. Type III-B $. \quad \Sigma^{+}=W^{+}=\mathrm{B}_{r}^{+}$,

$$
\tilde{\alpha}=e_{1}+e_{2}=\lambda_{1}+2 \lambda_{2}+\cdots+2 \lambda_{r} .
$$

A point $H \in \overline{P_{0}}$ is austere which is not totally geodesic if and only if $H=(1 / 2) H_{1}, H_{i}(2 \leq$ $i \leq r)$.

For each $H_{i}=(\pi / 4)\left(e_{1}+\cdots+e_{i}\right)$, we have $\tilde{\Sigma}_{H_{i}} \cong \mathrm{D}_{i} \oplus \mathrm{B}_{r-i}$. Therefore, by Proposition 7 and Theorem 5, if $i$ is even, then $K_{2} \exp \left(H_{i}\right) K_{1} \subset G, K_{2} \pi_{1}\left(\exp \left(H_{i}\right)\right) \subset$ $M_{1}, K_{1} \pi_{2}\left(\exp \left(H_{i}\right)\right) \subset M_{2}$ are weakly reflective for each $2 \leq i \leq r$. When $i$ is odd, since $-\mathrm{id}_{\mathfrak{a}} \notin W(\Sigma), H_{i}$ does not satisfy the sufficient condition in Theorem 5. Since $\operatorname{Span}\left(\tilde{\Sigma}_{H_{1}}\right) \neq \mathfrak{a}$, the point $(1 / 2) H_{1}$ does not satisfy the sufficient condition in Theorem 5 .

A compact symmetric triad whose symmetric triad is type III- $\mathrm{B}_{r}$ is one of the following: 
1. $(U \times U, \Delta(U \times U), \bar{K} \times \bar{K})$ where $(U, \bar{K})$ is a compact symmetric pair whose root system is type $\mathrm{B}_{r} \quad$ (condition (B)).

4.9. Type III-C $. \quad \Sigma^{+}=W^{+}=\mathrm{C}_{r}^{+}$,

$$
\tilde{\alpha}=2 e_{1}=2 \lambda_{1}+\cdots+2 \lambda_{r-1}+\lambda_{r} .
$$

If $m\left( \pm 2 e_{i}\right) \neq n\left( \pm 2 e_{i}\right)$, then a point $H \in \overline{P_{0}}$ is austere which is not totally geodesic if and only if $H=H_{i}(1 \leq i \leq r-1)$. If $m\left( \pm 2 e_{i}\right)=n\left( \pm 2 e_{i}\right)$, then $H \in \overline{P_{0}}$ is austere which is not totally geodesic if and only if $H=(1 / 2) H_{r}, H_{i}(1 \leq i \leq r-1)$. For each $H_{i}=$ $(\pi / 4)\left(e_{1}+\cdots+e_{i}\right)(1 \leq i \leq r-1)$, we have $\tilde{\Sigma}_{H_{i}} \cong \mathrm{C}_{i} \oplus \mathrm{C}_{r-i}$. Therefore, by Proposition 7 and Theorem 5, $K_{2} \exp \left(H_{i}\right) K_{1} \subset G, K_{2} \pi_{1}\left(\exp \left(H_{i}\right)\right) \subset M_{1}, K_{1} \pi_{2}\left(\exp \left(H_{i}\right)\right) \subset M_{2}$ are weakly reflective for each $1 \leq i \leq r-1$. Since $\operatorname{Span}\left(\tilde{\Sigma}_{(1 / 2) H_{r}}\right) \neq \mathfrak{a}$, the point $(1 / 2) H_{r}$ does not satisfy the sufficient condition in Theorem 5. A compact symmetric triad whose symmetric triad is type III- $\mathrm{C}_{r}$ is one of the following:

1. $(\mathrm{SU}(4 r), \mathrm{S}(\mathrm{U}(2 r) \times \mathrm{U}(2 r)), \mathrm{Sp}(2 r))$,

2. $(\mathrm{Sp}(2 r), \mathrm{U}(2 r), \mathrm{Sp}(r) \times \mathrm{Sp}(r))$,

3. $(U \times U, \Delta(U \times U), \bar{K} \times \bar{K})$ where $(U, \bar{K})$ is a compact symmetric pair whose root system is type $\mathrm{C}_{r} \quad$ (condition (B)).

4.10. Type III-BC $r . \quad \Sigma^{+}=W^{+}=\mathrm{BC}_{r}^{+}$,

$$
\tilde{\alpha}=2 e_{1}=2 \lambda_{1}+\cdots+2 \lambda_{r} .
$$

A point $H \in \overline{P_{0}}$ is austere which is not totally geodesic if and only if $H=H_{i}(1 \leq i \leq r)$. For each $H_{i}=(\pi / 4)\left(e_{1}+\cdots+e_{i}\right)(1 \leq i \leq r)$, we have $\tilde{\Sigma}_{H_{i}} \cong \mathrm{C}_{i} \oplus \mathrm{BC}_{r-i}$. Therefore, by Proposition 7 and Theorem 5, $K_{2} \exp \left(H_{i}\right) K_{1} \subset G, K_{2} \pi_{1}\left(\exp \left(H_{i}\right)\right) \subset M_{1}, K_{1} \pi_{2}\left(\exp \left(H_{i}\right)\right) \subset M_{2}$ are weakly reflective for each $1 \leq i \leq r$.

A compact symmetric triad whose symmetric triad is type III-BC $\mathrm{B}_{r}$ is one of the following:

1. $(\mathrm{SU}(2 r+2 s), \mathrm{S}(\mathrm{U}(2 r) \times \mathrm{U}(2 s)), \mathrm{Sp}(r+s)) \quad(r<s)$,

2. $(\mathrm{SU}(2(2 r+1)), \mathrm{S}(\mathrm{U}(2 r+1) \times \mathrm{U}(2 r+1)), \mathrm{Sp}(2 r+1)) \quad(1 \leq r)$,

3. $(\mathrm{Sp}(r+s), \mathrm{U}(r+s), \operatorname{Sp}(r) \times \mathrm{Sp}(s)) \quad(r<s)$,

4. $\left(E_{6}, \mathrm{SU}(6) \cdot \mathrm{SU}(2), F_{4}\right) \quad(r=1)$,

5. $\left(E_{6}, \mathrm{SO}(10) \cdot \mathrm{U}(1), F_{4}\right) \quad(r=1)$,

6. $\left(F_{4}, \mathrm{Sp}(3) \cdot \mathrm{Sp}(1), \mathrm{SO}(9)\right) \quad(r=1)$,

7. $(U \times U, \Delta(U \times U), \bar{K} \times \bar{K})$ where $(U, \bar{K})$ is a compact symmetric pair whose root system is type $\mathrm{BC}_{r} \quad$ (condition (B)).

4.11. Type III-D $. \quad \Sigma^{+}=W^{+}=\mathrm{D}_{r}^{+}$,

$$
\tilde{\alpha}=e_{1}+e_{2}=\lambda_{1}+2 \lambda_{2}+\cdots+2 \lambda_{r-2}+\lambda_{r-1}+\lambda_{r} .
$$

A point $H \in \overline{P_{0}}$ is austere which is not totally geodesic if and only if $H_{i}(2 \leq i \leq r-$ $1),(1 / 2) H_{1},(1 / 2) H_{r-1},(1 / 2) H_{r},(1 / 2)\left(H_{1}+H_{r-1}\right),(1 / 2)\left(H_{1}+H_{r}\right),(1 / 2)\left(H_{r-1}+H_{r}\right)$. 
For each $H_{i}=(\pi / 4)\left(e_{1}+\cdots+e_{i}\right)(2 \leq i \leq r-2)$, we have $\tilde{\Sigma}_{H_{i}} \cong \mathrm{D}_{i} \oplus \mathrm{D}_{r-i}$. Therefore, by Proposition 7 and Theorem 5, if $r$ and $i$ are even, then $K_{2} \exp \left(H_{i}\right) K_{1} \subset$ $G, K_{2} \pi_{1}\left(\exp \left(H_{i}\right)\right) \subset M_{1}, K_{1} \pi_{2}\left(\exp \left(H_{i}\right)\right) \subset M_{2}$ are weakly reflective for each $1 \leq$ $i \leq r$. When $H=H_{i}$ ( $i$ or $r$ is odd), $(1 / 2) H_{1},(1 / 2) H_{r-1},(1 / 2) H_{r},(1 / 2)\left(H_{1}+\right.$ $\left.H_{r-1}\right),(1 / 2)\left(H_{1}+H_{r}\right),(1 / 2)\left(H_{r-1}+H_{r}\right), H$ does not satisfy the sufficient condition in Theorem 5.

A compact symmetric triad whose symmetric triad is type III- $\mathrm{D}_{r}$ is one of the following:

1. $(U \times U, \Delta(U \times U), \bar{K} \times \bar{K})$ where $(U, \bar{K})$ is a compact symmetric pair whose root system is type $\mathrm{D}_{r} \quad$ (condition (B)).

4.12. Type III-E 6 . By Proposition $7,-\mathrm{id}_{\mathfrak{a}} \notin W(\tilde{\Sigma})$. Moreover, for each $H \in \mathfrak{a}$, $W\left(\tilde{\Sigma}_{H}\right) \subset W(\tilde{\Sigma})$ since $\tilde{\Sigma}_{H} \subset \tilde{\Sigma}$. Hence $-\operatorname{id}_{\mathfrak{a}} \notin W\left(\tilde{\Sigma}_{H}\right)$. Thus, each austere point does not satisfy the sufficient condition in Theorem 5 .

A compact symmetric triad whose symmetric triad is type III- $\mathrm{E}_{6}$ is one of the following:

1. $(U \times U, \Delta(U \times U), \bar{K} \times \bar{K})$ where $(U, \bar{K})$ is a compact symmetric pair whose root system is type $\mathrm{E}_{6} \quad$ (condition (B)).

4.13. Type III-E $. \quad \Sigma^{+}=W^{+}=\mathrm{E}_{7}^{+}, \Pi=\left\{\lambda_{1}, \lambda_{2}, \lambda_{3}, \lambda_{4}, \lambda_{5}, \lambda_{6}, \lambda_{7}\right\}$,

$$
\tilde{\alpha}=2 \lambda_{1}+2 \lambda_{2}+4 \lambda_{3}+4 \lambda_{4}+3 \lambda_{5}+2 \lambda_{6}+\lambda_{7} .
$$

A point $H \in \overline{P_{0}}$ is austere which is not totally geodesic if and only if $H=$ $H_{1}, H_{2}, H_{6},(1 / 2) H_{7}$. Since $\operatorname{Span}\left(\tilde{\Sigma}_{(1 / 2) H_{7}}\right) \neq \mathfrak{a}$, the point $(1 / 2) H_{7}$ does not satisfy the sufficient condition in Theorem 5.

(1) When $H=H_{1}$ We have $\Sigma_{H_{1}}^{+}=\Sigma^{+} \cap \operatorname{Span}_{\mathbf{Z}}\left\{\lambda_{2}, \lambda_{3}, \lambda_{4}, \lambda_{5}, \lambda_{6}, \lambda_{7}\right\}, W_{H_{1}}^{+}=\{\tilde{\alpha}\}$. Since $\left\langle\tilde{\alpha}, \lambda_{i}\right\rangle=0(2 \leq i \leq 7), \Sigma_{H_{1}} \perp W_{H_{1}}$. Hence, $\tilde{\Sigma}_{H_{1}}$ is isomorphic to $\Sigma_{H_{1}} \oplus W_{H_{1}}$ as a root system. Since $\left\{\lambda_{2}, \lambda_{3}, \lambda_{4}, \lambda_{5}, \lambda_{6}, \lambda_{7}\right\}$ is a fundamental system of $\Sigma_{H_{1}}$, we can see $\Sigma_{H_{1}} \cong \mathrm{D}_{6}$. Hence, we have $\tilde{\Sigma}_{H_{1}} \cong \mathrm{D}_{6} \oplus \mathrm{A}_{1}$. Therefore, by Proposition 7 and Theorem 5, $K_{2} \exp \left(H_{1}\right) K_{1} \subset G, K_{2} \pi_{1}\left(\exp \left(H_{1}\right)\right) \subset M_{1}, K_{1} \pi_{2}\left(\exp \left(H_{1}\right)\right) \subset M_{2}$ are weakly reflective.

(2) When $H=H_{2} \quad$ We have

$$
\begin{aligned}
\Sigma_{H_{2}}^{+}= & \Sigma^{+} \cap \operatorname{Span}_{\mathbf{Z}}\left\{\lambda_{1}, \lambda_{3}, \lambda_{4}, \lambda_{5}, \lambda_{6}, \lambda_{7}\right\}, \\
W_{H_{2}}= & \left\{\lambda_{0}, \lambda_{0}+\lambda_{7}, \lambda_{0}+\lambda_{6}+\lambda_{7}, \lambda_{0}+\lambda_{5}+\lambda_{6}+\lambda_{7}, \lambda_{0}+\lambda_{4}+\lambda_{5}+\lambda_{6}+\lambda_{7},\right. \\
& \left.\lambda_{0}+\lambda_{3}+\lambda_{4}+\lambda_{5}+\lambda_{6}+\lambda_{7}, \lambda_{0}+\lambda_{1}+\lambda_{3}+\lambda_{4}+\lambda_{5}+\lambda_{6}+\lambda_{7}\right\},
\end{aligned}
$$

where $\lambda_{0}=\lambda_{1}+2 \lambda_{2}+2 \lambda_{3}+3 \lambda_{4}+2 \lambda_{5}+\lambda_{6}$. Hence,

$$
\Pi_{H_{2}}:=\left\{\lambda_{0}, \lambda_{1}, \lambda_{3}, \lambda_{4}, \lambda_{5}, \lambda_{6}, \lambda_{7}\right\}
$$

is a fundamental system of $\tilde{\Sigma}_{H_{2}}$. For $i=1,3 \leq i \leq 6$, we have $\left\langle\lambda_{0}, \lambda_{i}\right\rangle=0,\left\langle\lambda_{0}, \lambda_{7}\right\rangle=$ $\left\langle\lambda_{6}, \lambda_{7}\right\rangle$. Thus, $\Pi_{H_{2}}$ corresponds to the Dynkin diagram of type $A_{7}$. Therefore, we obtain 
$\tilde{\Sigma}_{H_{2}} \cong A_{7}$. By Proposition 7, we have $-\mathrm{id}_{\mathfrak{a}} \notin W\left(\tilde{\Sigma}_{H_{2}}\right)$. Thus, $H_{2}$ does not satisfy the sufficient condition in Theorem 5.

(3) When $H=H_{6} \quad$ Similarly, we set $\lambda_{0}=\lambda_{2}+\lambda_{3}+2 \lambda_{4}+2 \lambda_{5}+2 \lambda_{6}+\lambda_{7}$. Then, the set

$$
\Pi_{H_{6}}=\left\{\lambda_{0}, \lambda_{1}, \lambda_{2}, \lambda_{3}, \lambda_{4}, \lambda_{5}, \lambda_{7}\right\}
$$

is a fundamental system of $\tilde{\Sigma}_{H_{6}}$. For $2 \leq i \leq 5, i=7$, we have $\left\langle\lambda_{0}, \lambda_{i}\right\rangle=0,\left\langle\lambda_{0}, \lambda_{1}\right\rangle=$ $\left\langle\lambda_{1}, \lambda_{3}\right\rangle$. The set $\Pi_{H_{6}}$ corresponds to the Dynkin diagram of type $\mathrm{D}_{6} \oplus \mathrm{A}_{1}$. Thus, we have $\tilde{\Sigma}_{H_{6}} \cong \mathrm{D}_{6} \oplus \mathrm{A}_{1}$. Therefore, by Proposition 7 and Theorem $5, K_{2} \exp \left(H_{6}\right) K_{1} \subset$ $G, K_{2} \pi_{1}\left(\exp \left(H_{6}\right)\right) \subset M_{1}$ and $K_{1} \pi_{2}\left(\exp \left(H_{6}\right)\right) \subset M_{2}$ are weakly reflective.

A compact symmetric triad whose symmetric triad is type III-E $\mathrm{E}_{7}$ is one of the following:

1. $(U \times U, \Delta(U \times U), \bar{K} \times \bar{K})$ where $(U, \bar{K})$ is a compact symmetric pair whose root system is type $\mathrm{E}_{7} \quad$ (condition (B)).

4.14. Type III-E $. \quad \Sigma^{+}=W^{+}=\mathrm{E}_{8}^{+}, \Pi=\left\{\lambda_{1}, \lambda_{2}, \lambda_{3}, \lambda_{4}, \lambda_{5}, \lambda_{6}, \lambda_{7}, \lambda_{8}\right\}, \tilde{\alpha}=$ $2 \lambda_{1}+3 \lambda_{2}+4 \lambda_{3}+6 \lambda_{4}+5 \lambda_{5}+4 \lambda_{6}+3 \lambda_{7}+2 \lambda_{8}$. A point $H \in \overline{P_{0}}$ is austere which is not totally geodesic if and only if $H=H_{1}, H_{8}$.

(1) When $H=H_{1}$ We set $\lambda_{0}=2 \lambda_{1}+2 \lambda_{2}+3 \lambda_{3}+4 \lambda_{4}+3 \lambda_{5}+2 \lambda_{6}+\lambda_{7}$. Then, the set $\Pi_{H_{1}}=\left\{\lambda_{0}, \lambda_{2}, \lambda_{3}, \lambda_{4}, \lambda_{5}, \lambda_{6}, \lambda_{7}, \lambda_{8}\right\}$ is a fundamental system of $\tilde{\Sigma}_{H_{1}}$. For each $2 \leq i \leq 7$, we have $\left\langle\lambda_{0}, \lambda_{i}\right\rangle=0,\left\langle\lambda_{0}, \lambda_{8}\right\rangle=\left\langle\lambda_{7}, \lambda_{8}\right\rangle$. Thus $\Pi_{H_{1}}$ corresponds to the Dynkin diagram of type $\mathrm{D}_{8}$. Hence, $\tilde{\Sigma}_{H_{1}} \cong \mathrm{D}_{8}$. Therefore, by Proposition 7 and Theorem 5, we have $K_{2} \exp \left(H_{1}\right) K_{1} \subset G, K_{2} \pi_{1}\left(\exp \left(H_{1}\right)\right) \subset M_{1}, K_{1} \pi_{2}\left(\exp \left(H_{1}\right)\right) \subset M_{2}$ are weakly reflective.

(2) When $H=H_{8} \quad$ We have $\Sigma_{H_{8}}^{+}=\Sigma^{+} \cap \operatorname{Span}_{\mathbf{Z}}\left\{\lambda_{1}, \lambda_{2}, \lambda_{3}, \lambda_{4}, \lambda_{5}, \lambda_{6}, \lambda_{7}\right\}, W_{H_{8}}=\{\tilde{\alpha}\}$. For each $1 \leq i \leq 7$, we have $\left\langle\tilde{\alpha}, \lambda_{i}\right\rangle=0$. Thus, $\Sigma_{H_{8}} \perp W_{H_{8}}$. Hence $\tilde{\Sigma}_{H_{8}}$ is isomorphic to $\tilde{\Sigma}_{H_{8}} \cong \Sigma_{H_{8}} \oplus W_{H_{8}}$ as a root system. Since the set $\left\{\lambda_{1}, \lambda_{2}, \lambda_{3}, \lambda_{4}, \lambda_{5}, \lambda_{6}, \lambda_{7}\right\}$ is a fundamental system of $\Sigma_{H_{8}}$, we can see that $\Sigma_{H_{8}} \cong \mathrm{E}_{7}$. Thus, $\tilde{\Sigma}_{H_{8}} \cong \mathrm{E}_{7} \oplus \mathrm{A}_{1}$. Therefore, by Proposition 7 and Theorem 5, $K_{2} \exp \left(H_{8}\right) K_{1} \subset G, K_{2} \pi_{1}\left(\exp \left(H_{8}\right)\right) \subset M_{1}, K_{1} \pi_{2}\left(\exp \left(H_{8}\right)\right) \subset M_{2}$ are weakly reflective.

A compact symmetric triad whose symmetric triad is type III-E $\mathrm{E}_{8}$ is one of the following:

1. $(U \times U, \Delta(U \times U), \bar{K} \times \bar{K})$ where $(U, \bar{K})$ is a compact symmetric pair whose root system is type $\mathrm{E}_{8} \quad$ (condition (B)).

4.15. Type III-F $. \quad \Sigma^{+}=W^{+}=\mathrm{F}_{4}^{+}, \Pi=\left\{\lambda_{1}=e_{2}-e_{3}, \lambda_{2}=e_{3}-e_{4}, \lambda_{3}=\right.$ $\left.e_{4}, \lambda_{4}=(1 / 2)\left(e_{1}-e_{2}-e_{3}-e_{4}\right)\right\}, \tilde{\alpha}=e_{1}+e_{2}=2 \lambda_{1}+3 \lambda_{2}+4 \lambda_{3}+2 \lambda_{4}$. A point $H \in \overline{P_{0}}$ is austere which is not totally geodesic if and only if $H=H_{1}=(\pi / 4)\left(e_{1}+e_{2}\right), H_{4}=(\pi / 2) e_{1}$. 
(1) When $H=H_{1}$ We have $\tilde{\Sigma}_{H_{1}} \cong \mathrm{C}_{4}$. Therefore, by Proposition 7 and Theorem 5, $K_{2} \exp \left(H_{1}\right) K_{1} \subset G, K_{2} \pi_{1}\left(\exp \left(H_{1}\right)\right) \subset M_{1}, K_{1} \pi_{2}\left(\exp \left(H_{1}\right)\right) \subset M_{2}$ are weakly reflective.

(2) When $H=H_{4}$ We have $\tilde{\Sigma}_{H_{4}} \cong B_{4}$. Therefore, by Proposition 7 and Theorem 5, $K_{2} \exp \left(H_{4}\right) K_{1} \subset G, K_{2} \pi_{1}\left(\exp \left(H_{4}\right)\right) \subset M_{1}, K_{1} \pi_{2}\left(\exp \left(H_{4}\right)\right) \subset M_{2}$ are weakly reflective.

A compact symmetric triad whose symmetric triad is type III-F 4 is one of the following:

1. $(U \times U, \Delta(U \times U), \bar{K} \times \bar{K})$ where $(U, \bar{K})$ is a compact symmetric pair whose root system is type $\mathrm{F}_{4} \quad$ (condition (B)).

4.16. Type III-G $. \quad \Sigma^{+}=W^{+}=\mathrm{G}_{2}^{+}, \Pi=\left\{\lambda_{1}=e_{1}-e_{2}, \lambda_{2}=-2 e_{1}-e_{2}+e_{3}\right\}$, $\tilde{\alpha}=-e_{1}-e_{2}+2 e_{3}=3 \lambda_{1}+2 \lambda_{2}$.

A point $H \in \overline{P_{0}}$ is austere which is not totally geodesic if and only if $H=H_{2}=$ $(\pi / 12)\left(-e_{1}-e_{2}+2 e_{3}\right)=(\pi / 12)\left(3 \lambda_{1}+2 \lambda_{2}\right)$. We have $\Sigma_{H_{2}}^{+}=\left\{\lambda_{1}\right\}, W_{H_{2}}^{+}=\left\{3 \lambda_{1}+2 \lambda_{2}\right\}$. Thus, $\tilde{\Sigma}_{H_{2}}^{+}=\left\{\lambda_{1}, 3 \lambda_{1}+2 \lambda_{2}\right\}$ Therefore, by Proposition 7 and Theorem 5, $K_{2} \exp \left(H_{2}\right) K_{1} \subset$ $G, K_{2} \pi_{1}\left(\exp \left(H_{2}\right)\right) \subset M_{1}, K_{1} \pi_{2}\left(\exp \left(H_{2}\right)\right) \subset M_{2}$ are weakly reflective.

A compact symmetric triad whose symmetric triad is type III- $\mathrm{G}_{2}$ is one of the following:

1. $(U \times U, \Delta(U \times U), \bar{K} \times \bar{K})$ where $(U, \bar{K})$ is a compact symmetric pair whose root system is type $\mathrm{G}_{2} \quad$ (condition (B)).

\section{References}

[1] N. Bourbaki, Groupes et algebres de Lie, Hermann, Paris, 1975.

[2 ] O. Goertsches and G. Thorbergsson, On the Geometry of the orbits of Hermann action, Geom. Dedicata 129 (2007), 101-118.

[ 3 ] R. Harvey and H. B. Lawson, JR., Calibrated geometries, Acta Math. 148 (1982), 47-157.

[ 4 ] O. IKAWA, The geometry of symmetric triad and orbit spaces of Hermann actions, J. Math. Soc. Japan 63 (2011), 79-136.

[ 5 ] O. IKAWA, A note on symmetric triad and Hermann actions, Proceedings of the workshop on differential geometry of submanifolds and its related topics, Saga, August 4-6 (2012), 220-229.

[6] O. IKAWA, T. SAKAI and H. TASAKI, Weakly reflective submanifolds and austere submanifolds, J. Math. Soc. Japan 61 (2009), 437-481.

[ 7 ] A. Kollross, A classification of hyperpolar and cohomogeneity one actions, Trans. Amer. Math. Soc. 354, no. 2 (2002), 571-612.

[ 8 ] S. Dominic and P. LeUng, The reflection principle for minimal submanifolds of Riemannian symmetric spaces, J. Differential Geom. 8 (1973), 153-160.

[ 9 ] T. Matsuki, Double coset decompositions of reductive Lie groups arising from two involutions, J. Algebra 197 (1997), 49-91.

[10] J. TITs, Classification of algebraic semisimple groups, Algebraic groups and discontinuous subgroups (Proc. Sympos. Pure Math. Boulder, Colo., 1965), Amer. Math. Soc. (1966), 33-62. 
Present Address:

Osaka City University Advanced Mathematical Institute (OCAMI), 3-3-138 SUGIMOTO, SUMIYOSHI-KU, OSAKA 558-8585, JAPAN.

e-mail: kudamono.shinji@gmail.com 